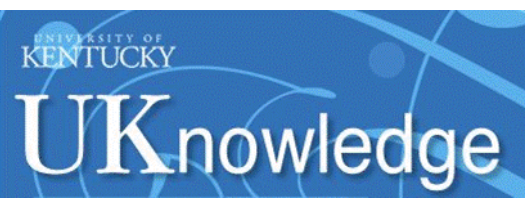

University of Kentucky

UKnowledge

\title{
THE DEVELOPMENT OF EXPERT FACE PROCESSING: ARE INFANTS SENSITIVE TO NORMAL DIFFERENCES IN SECOND- ORDER RELATIONAL INFORMATION?
}

Angela Hayden

University of Kentucky, angiehayds@hotmail.com

Right click to open a feedback form in a new tab to let us know how this document benefits you.

\section{Recommended Citation}

Hayden, Angela, "THE DEVELOPMENT OF EXPERT FACE PROCESSING: ARE INFANTS SENSITIVE TO NORMAL DIFFERENCES IN SECOND-ORDER RELATIONAL INFORMATION?" (2006). University of Kentucky Master's Theses. 403.

https://uknowledge.uky.edu/gradschool_theses/403

This Thesis is brought to you for free and open access by the Graduate School at UKnowledge. It has been accepted for inclusion in University of Kentucky Master's Theses by an authorized administrator of UKnowledge. For more information, please contact UKnowledge@lsv.uky.edu. 


\section{ABSTRACT OF THESIS}

\section{THE DEVELOPMENT OF EXPERT FACE PROCESSING: ARE INFANTS SENSITIVE TO NORMAL DIFFERENCES IN SECOND-ORDER RELATIONAL INFORMATION?}

Diamond and Carey (1986) identify sensitivity to second-order relational information (i.e., spatial relations among features such as the distance between eyes) as a vital part of achieving expertise with face processing. Previous research suggests that 5-month-olds are sensitive to second-order relational information when shown line drawings of faces in which this information has been manipulated to an exaggerated degree. The present series of experiments explored infants' sensitivity to second-order relational information using photographs of real faces and with second-order manipulations that were within the normal range of human variability. A discrimination study conducted with adults provided additional evidence that the second-order manipulations were within the normal range. Five- and 7- month-olds exhibited sensitivity to changes in second-order relational information. Moreover, 5-months detected second-order changes in upright but not in inverted faces, thereby exhibiting an inversion effect that has been considered to be a hallmark of second-order relational processing in adulthood. These results suggest that infants as young as 5 months of age are sensitive to second-order relational changes that are within the normal range of human variability. They also indicate that at least rudimentary aspects of face-processing expertise are available early in life.

KEYWORDS: Infant Development, Face Perception, Relational Information, Sensitivity, Cognitive Discrimination 
Angela Hayden

December 11, 2006 
THE DEVELOPMENT OF EXPERT FACE PROCESSING: ARE INFANTS SENSITIVE TO NORMAL DIFFERENCES IN SECOND-ORDER RELATIONAL INFORMATION?

By

Angela Hayden

Ramesh Bhatt, Ph.D.

Director of Thesis

David Berry, Ph.D.

Director of Graduate Studies

December 11, 2006 


\section{RULES FOR THE USE OF THESES}

Unpublished theses submitted for the Master's degree and deposited in the University of Kentucky Library are as a rule open for inspection, but are to be used only with due regard to the rights of the authors. Bibliographical references may be noted, but quotations or summaries of parts may be published only with the permission of the author and with the usual scholarly acknowledgements.

Extensive copying or publication of the thesis in whole or in part also requires the consent of the Dean of the Graduate School of the University of Kentucky. 


\section{THESIS}

Angela Hayden

The Graduate School

University of Kentucky

2006 
THE DEVELOPMENT OF EXPERT FACE PROCESSING: ARE INFANTS SENSITIVE TO NORMAL DIFFERENCES IN SECOND-ORDER RELATIONAL INFORMATION?

\title{
THESIS
}

A thesis submitted in partial fulfillment of the requirements for the degree of Master of Arts in the College of Arts and Sciences at the University of Kentucky

By

\author{
Angela Hayden \\ Lexington, Kentucky
}

Director: Dr. Ramesh Bhatt, Professor of Psychology

2006

Copyright $@$ Angela Hayden 2006 


\section{MASTER'S THESIS RELEASE}

I authorize the University of Kentucky Libraries to reproduce this thesis in whole or in part for purposes of research.

Angela Hayden

December 11, 2006 


\section{ACKNOWLEDGEMENTS}

While this thesis was ultimately an individual work, I benefited greatly from the guidance and support of my advisor, Dr. Ramesh Bhatt. Your insight and input was invaluable to the completion of this thesis. You are a wonderful teacher and a great role model for anyone aspiring to enter the academic world. I would also like to thank the rest of the members of my thesis committee: Dr. Jane Joseph and Dr. Elizabeth Lorch. Your critical analysis of my proposal was vital to the direction and the success of my thesis. Your comments and suggestions were also extremely helpful in revising my thesis.

Finally, I would like to thank my parents, Michael and Patricia Hayden. You have always supported me in my endeavors and I appreciate that greatly. I wouldn't be the person I am today without your love and encouragement. 


\section{TABLE OF CONTENTS}

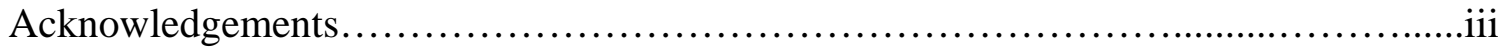

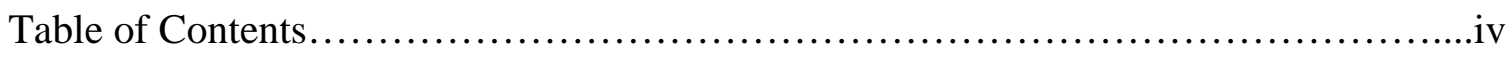

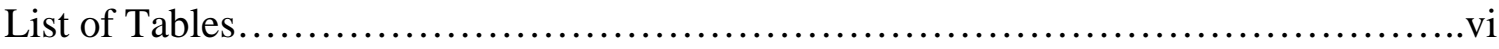

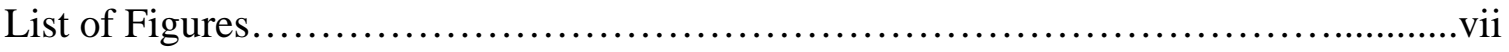

List of Files................................................................................

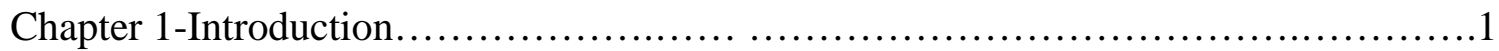

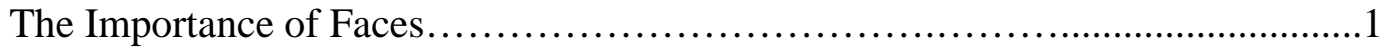

Development of Different Kinds of Facial Processing...............................1

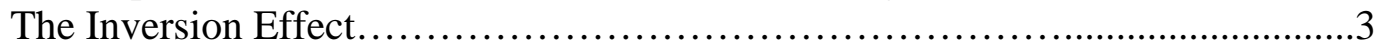

Developmental Aspects of Face Processing in Childhood...............................

Sensitivity to Second-Order Relational Information in Infancy....................5

The Present Study........................................................

Chapter 2-Experiment 1 .........................................................

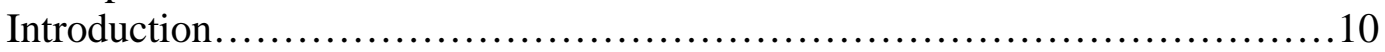

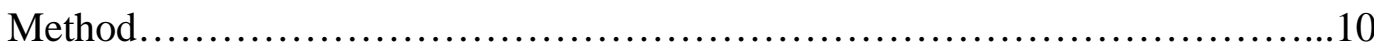

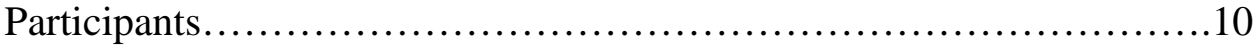

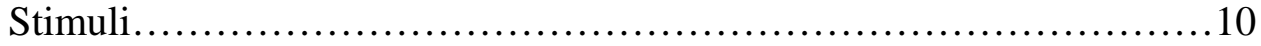

Apparatus..........................................................

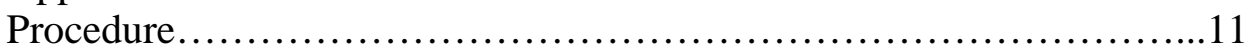

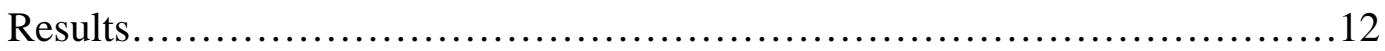

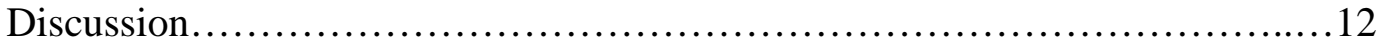

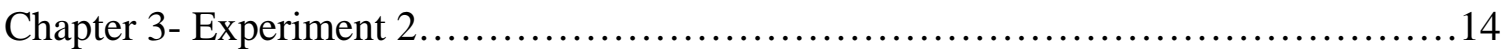

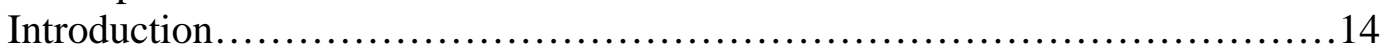

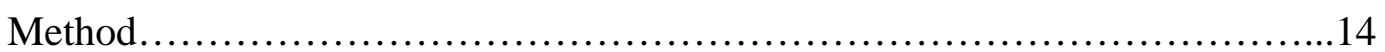

Participants..........................................................

Stimuli........................................................... 14

Apparatus............................................................

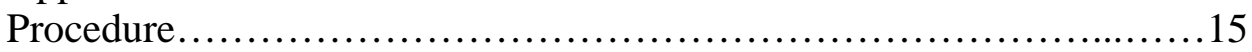

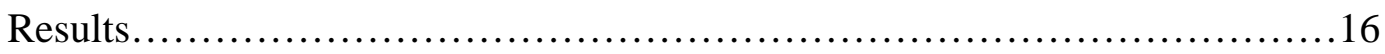

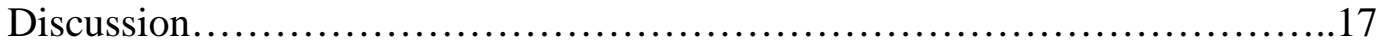

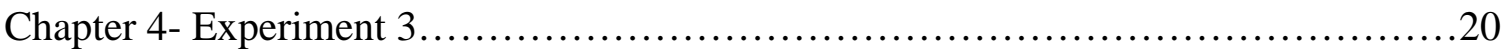

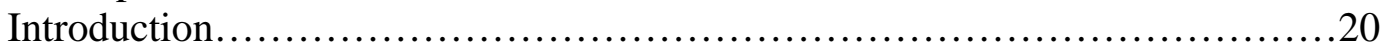

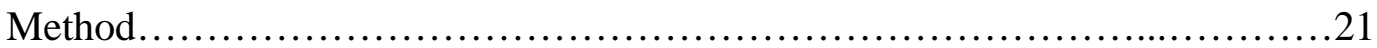

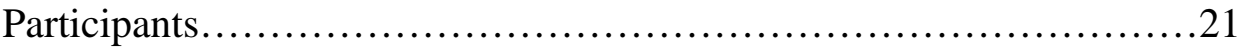

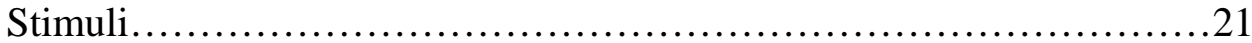

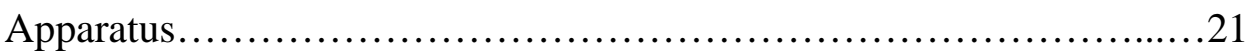

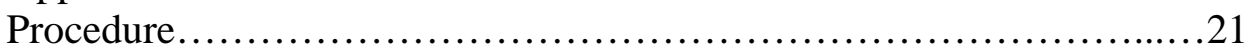

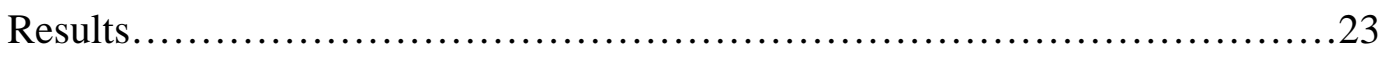

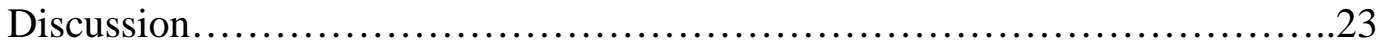

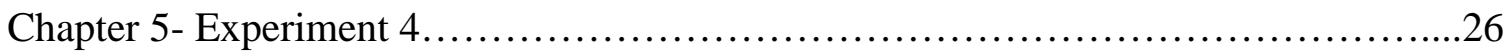




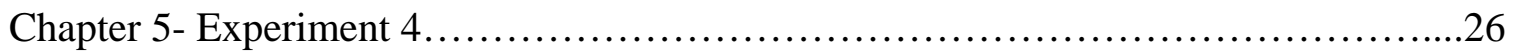

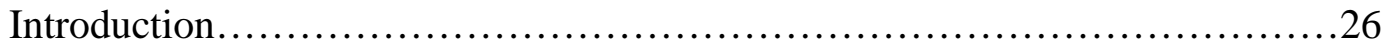

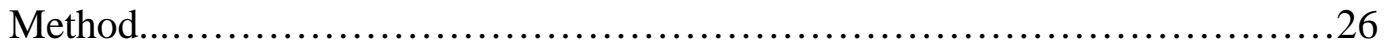

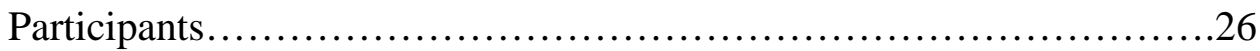

Stimuli.....................................................26

Apparatus.........................................................26

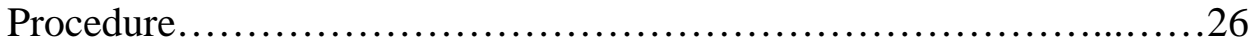

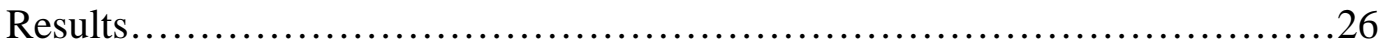

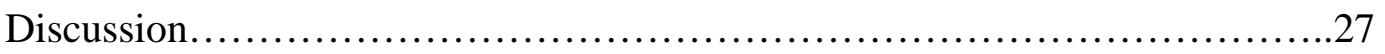

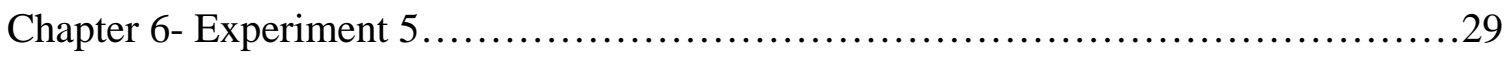

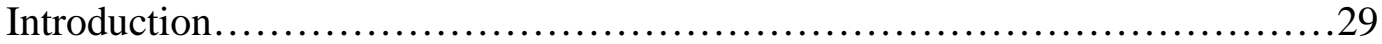

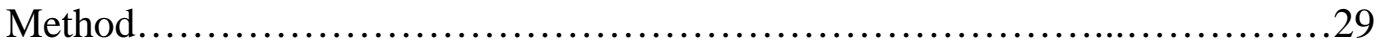

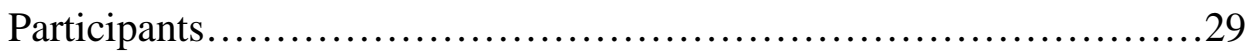

Stimuli......................................................... 30

Procedure....................................................... 30

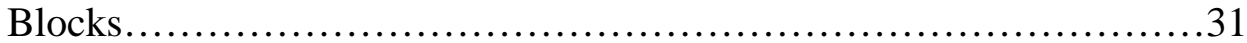

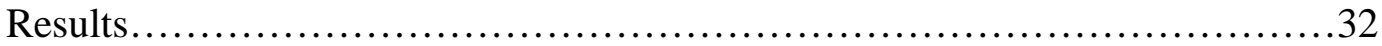

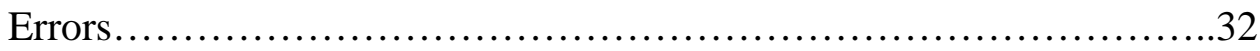

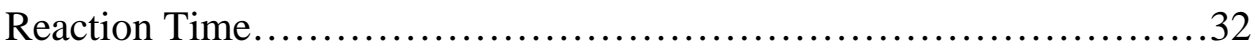

Discussion..............................................................

Chapter 7- General Discussion.................................................37

Evidence of Second-Order Relational Manipulation Within Normal Range......37

Comparison to Other Studies and Farkas Measurements.................37

The Inversion Effect..............................................37

Relationship of the Present Study to Previous Infant and Adult Studies...........39

Valentine's 'Face-Space' Hypothesis..........................................40

The Uniqueness of Face Processing......................................41

Future Studies...........................................................42

References.................................................................... 44

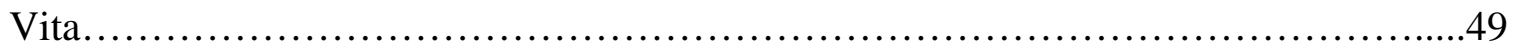




\section{LIST OF TABLES}

Table 2.1, Mean Preference Score (percentage) for the Normal Face During the Spontaneous Preference Test Trials of Experiment 1.

Table 3.1, Mean Fixation Times (seconds) During the Habituation Trials and Preference Scores (percentages) for the Novel Face During the Test Trials of Experiment 2.

Table 4.1, Mean (and Standard Deviation) of Percent Preference to the Non-Moving Upright Face for 7-Month-Olds in Experiment 3.

Table 5.1, Mean (and Standard Deviation) of Percent Preference to the Non-Moving face (in both the upright and the inverted conditions) for 5-Month-Olds in Experiment 4.

Table 7.1, Overview of all studies. 


\section{LIST OF FIGURES}

Figure 6.1, Graph of mean error rate for the Jenny and Pam faces for upright and inverted trials. Figure 6.2, Graphs of individual error data for adults. The top graph shows errors made in response to the Jenny face. The bottom graph shows errors made in response to the Pam face. Participants 1 through 5 saw the Jenny face initially, followed by the Pam face. Participants 6 through 10 saw the Pam face initially, followed by the Jenny face

Figure 6.3, Graph of mean reaction time (log transformed) to the Jenny and Pam faces for upright and inverted trials. 
LIST OF FILES

1. Masters.pdf 


\section{Chapter 1}

\section{Introduction}

There has been great controversy in the literature as to whether faces are unique in the perceptual system. This controversy stems from research showing that newborns track face-like patterns further than non-face-like patterns (Goren, Sarty, \& Wu, 1975), as well as several studies showing that infants prefer their mother’s face over a stranger's face (e.g., Pascalis, de Schonen, Morton, Deruelle, \& Fabre-Grenet, 1995; Walton, Bower, \& Bower, 1992). There are many differing theories on the apparent uniqueness of faces. Some (Farah, Rabinowitz, Quinn, \& Liu, 2000; Kanwisher, McDermott, \& Chun, 1997) posit that there are mechanisms in the brain solely for the purpose of processing faces. Jiang, Rosen, Zeffiro, VanMeter, Blanz, \& Riesenhuber (2006) proposed a shape-based model of face processing in which neurons in the Fusiform Face Area (FFA) are tuned to face-like stimuli. In their model, the neurons that are tuned to detect facial stimuli are sensitive enough to respond to a familiar face, but broadly tuned enough to respond to an unfamiliar face as being within the 'category' of faces. Others (Johnson \& Morton, 1991; de Schonen \& Mathivet, 1989) assume that perceptual constraints attract infants to faces and later develop into a domain-specific cognitive structure that processes faces. Turati (2004) proposed a model in which infants' attraction to faces is due to 'nonspecific perceptual constraints' that attract infants to object properties such as up-down asymmetry (newborns prefer images that have more elements at the top over those that have more elements on the bottom) as well as congruency (newborns prefer that the outer features and inner features of an object be the same shape). Most of these theories allow for development in faceprocessing that leads to expertise.

\section{Development of Different Kinds of Facial Processing}

In addition to the theories on when specific processing of faces develops, there are also many theories about the development of the capacity to process different kinds of facial information. Diamond and Carey (1977) proposed that adults employ two categorically different types of information in face processing: featural information and relational information. Featurebased processing occurs when the features of the face (e.g., a single eye, or the mouth) can be processed individually apart from the face as a whole (Diamond \& Carey, 1986). Most objects are identified by this parts-based information. However, relational information among features or parts is also a component of object representations, at least in some cases. Not all researchers 
agree that featural and relational information are two categorically different types of information. Jiang et al. (2006) suggested that these two types of information are quantitatively, but not qualitatively, different. Below the perceptual level at which faces are identified as faces (at the C2 level), Jiang et al. found that adults respond to featural and relational changes in the same fashion: as changes in stimulus shape. It is at this point unclear whether featural and configural information are two categorically different types of information. These types of information are, however, useful for determining the skill with which someone can discriminate between two faces: using relational information, at least a specific type of relational information, is an indication of the capacity for expertise with faces.

Diamond and Carey argued that two specific types of relational information are involved in face processing: first-order and second-order relational processing. First-order relational processing involves using the gross (categorical) spatial relationships between the parts of an object to match the object with its configural prototype. Seeing a pair of eyes above a nose, and a nose in turn above a mouth, are examples of first-order pieces of information that people use in order to identify a face from a non-face. First-order relational processing serves as an indicator of whether the arrangement of an object differs from the prototypical arrangement for that class of objects. Second-order relational processing involves using the fine (metric) spatial distances between features in order to identify one form from another within a particular category of objects. For example, people vary in the distance between their eyes, between the nose and the mouth, and so on. Part of identifying someone's face efficiently involves featural processing as well as both types of relational processing (first-order and second-order), and research indicates that adult expertise in facial processing originates from the ability to use second-order processing.

According to Diamond \& Carey (1986), relational processing is generally utilized with categories of objects with which a person has expertise. For many, relational processing is used solely for face processing because faces are the only objects that that they have had enough experience with to employ this type of processing. Dog experts and car experts are two groups of people that have been found to utilize relational processing to identify dogs and cars, respectively (Diamond \& Carey, 1986; Gauthier \& Curby, 2005). Interestingly, car experts show activation in the cortical area that was thought to be specific to face processing (Fusiform Face Area) when asked to distinguish between two types of cars (Gauthier \& Curby, 2005). This 
suggests that the Fusiform Face Area is not necessarily specific to faces, but is instead is used to process objects in one’s area of expertise.

\section{The Inversion Effect}

Many researchers (including Diamond \& Carey, 1977) use the inversion effect as evidence that relational processing is categorically different from featural processing. Inverting a face keeps the featural processing of the face fairly intact, (Freire, Lee, \& Symons, 2000; Mondloch, LeGrand, \& Maurer, 2002; Mondloch, Dobson, Parsons, \& Maurer, 2004; Searcy \& Bartlett, 2003) although it disrupts it to some extent in some studies (Moscovitch \& Moscovitch, 2000) and to a great extent in two recently-published studies (Riesenhuber, Jarudi, Gilad, \& Sinha, 2004; Yovel \& Duchaine, 2006). In the majority of studies, however, inversion disrupts second-order relational information processing more than featural information (Itier \& Taylor, 2004). Adults perform more poorly when discriminating between two inverted faces than discriminating between two upright faces (Diamond \& Carey, 1977; Freire et al., 2000; Goldstein \& Chance, 1980; McKone, 2004; Yin 1969) when the faces differ only in second-order relational information (Freire et al., 2000).

When viewing upright and inverted faces during event-related potential (ERP) studies, the component that is specific to face processing, the N170, exhibits characteristics that parallel the results of behavioral studies: the N170 response latency is increased and the amplitude enhanced for inverted faces as compared to upright faces (Bentin, Allison, Puce, Perez, \& McCarthy, 1996; George, Evans, Fiori, Davidoff, \& Renault, 1996), showing that there is a definitive difference between the processing of upright and inverted faces, even in terms of neurological indicators.

The inversion effect also suggests evidence of expertise with faces (Diamond \& Carey, 1986). Subjects show the inversion effect for faces, but not for other objects such as airplanes, houses, bridges (Yin, 1969), and landscapes (Diamond \& Carey, 1986). In the dog expertise study described above (Diamond \& Carey, 1986), 'dog novices’ did not show evidence of the inversion effect, but 'dog experts' did. Based on these results, Diamond and Carey concluded that canonical orientation is important for discrimination of categories of objects on which one is an expert. 


\section{Developmental Aspects of Face Processing in Childhood}

There is a great amount of debate among researchers in regard to the development of second-order relational processing. Some studies suggest that young children rely more on features than relational information in processing faces. These studies show an increasing reliance on relational processing (Schwartzer, 2002) for upright faces until approximately 10 to 14 years of age (Carey \& Diamond, 1994; Hay \& Cox, 2000; Itier \& Taylor, 2004; Mondloch et al., 2002, 2004). According to these studies, with increasing age, children show an escalating ability to discriminate faces that are upright based on both second-order information alone (using the same face throughout the experiment and manipulating metric relationships between features; e.g., Mondloch et al., 2002, 2004) as well as combined second-order and featural information (using an entirely new, unfamiliar face to test discrimination; e.g., Carey and Diamond, 1994; Hay \& Cox, 2000). In most of these studies, there is also an increase in the inversion effect with age (Carey and Diamond, 1994; Hay and Cox, 2000; Mondloch et al., 2002, 2004). The results described above illustrate an increasing expertise with faces; specifically, an increasing ability to use second-order relational information to identify faces.

Mondloch, Leis, \& Maurer (2006) suggest that that the ability to detect second-order relational information begins at approximately 5-6 years of age and becomes more refined after that. In this study, 4-year-olds did not even exhibit sensitivity to second-order information. In contrast, other recent studies have demonstrated that not only are 4-year-olds sensitive to relational information, but also that there is no increase in the ability to use second-order relational information with age, at least from four years to adulthood (McKone \& Boyer, 2006; Pellicano, Rhodes, \& Peters, 2006). For instance, Pellicano et al. found that 4-year-olds show adult-like levels of the inversion effect and concluded that adult-like sensitivity to second-order relational processing is available by 4 years of age. The authors of these studies speculated that previous studies had not come to these same conclusions because of methodological issues. Thus, there is uncertainty in the literature concerning the nature of development of second-order information processing.

While it is debatable whether or not the ability to use second-order relational information increases with age and exposure to increasing numbers of faces, most studies suggest that sensitivity to this information is present in childhood. This is exhibited by the fact that, in most studies that show that there is a developmental change during childhood in relational processing, 
the ability to distinguish between two faces that differ only in second-order relational information is not entirely absent, even at very young ages (e.g., Hay \& Cox, 2000; Mondloch et al., 2002, 2004). Younger children in these studies may be inferior to adults in the use of this information, but, in general, their levels of discrimination between faces that differ only in second-order information are above chance. As noted above, Mondloch et al. (2006) is an exception to these findings; in this study, 4-year-olds failed to discriminate between an unaltered face and a face that had been manipulated based on second-order relational information.

Sensitivity to Second-Order Relational Information in Infancy

Evidence is also growing that infants are sensitive to relational information in upright faces [both first-order and second-order relational information, in terms of the Diamond and Carey (1986) model] (Bertin \& Bhatt, 2004; Bhatt, Bertin, Hayden, \& Reed 2005; Rose, Jankowski, \& Feldman, 2002; Schwarzer \& Zauner, 2003; Thompson, Madrid, Westbrook, \& Johnston, 2001). Recall that, according to Diamond and Carey (1994), the ability to detect second-order relational information is necessary in order to begin the process of gaining expertise in face processing. Therefore, if infants are sensitive to second-order relational information, then very early in life they have the tools necessary to become experts at face processing.

Rose et al. (2002) and Thompson et al. (2001) suggest that infants as young as 7 months of age may be sensitive to relational information. Seven-month-old infants in the Rose et al. study were able to extract enough relational information from habituation to a single photograph of a face (when repeatedly paired with photographs of novel faces) to identify the same face in a $3 / 4$ view. The results of this study suggest that 7 -month-old infants responded as though the relationships among parts of the face are the same whether seen full-on or at a $3 / 4$ profile. However, infants in this study also had the advantage of being able to use featural information in discrimination, as the novel face that infants saw was a new face with both different relational information as well as different featural information. Thus, whether or not infants were actually responding to second-order information in these faces is an open question.

Seven-month-olds in the Thompson et al. (2001) study, when shown paired faces over 18 trials, preferred a more prototypical face than the same face in which the second-order properties (the distances between the features) had been distorted to make the face appear abnormal. However, one drawback to the Thompson et al. study is that, in order to change the second-order 
relationships between the features of the face stimuli used, Thompson et al. added to or subtracted sections from the faces. Under these circumstances, not only were the experimenters altering the second-order information, they were also altering the featural information in the faces, as the face frame is considered a feature of the face. In essence, Thompson et al. were elongating or shortening the frame of these faces, thus altering the featural information in the face.

Bertin and Bhatt (2004) examined configural processing by 6-month-olds using the Thatcher Illusion. The Thatcher Illusion is produced by inverting the eyes as well as the mouth of a face while keeping the rest of the face upright. This manipulation keeps the featural information of the face intact (because the actual features are not distorted-the eyes and the mouth remain the same shape and color). It also keeps the first-order relational information intact by maintaining the 'facedness' (eyes above nose, nose above mouth) of the face. Secondorder relational information, however, is disrupted because the metric distances between the features are distorted. The end result is a face that most children and adults find grotesque when upright and relatively normal when inverted (Bartlett \& Searcy, 1993; Lewis, 2003). Some experimenters cite this inversion effect to argue that this manipulation affects purely secondorder relational processing (e.g. Bartlett \& Searcy, 1993). Bertin and Bhatt (2004) found that 6month-olds, like children and adults, could detect thatcherized faces when they were upright but not when they were inverted.

Bhatt et al. (2005) explored this area further with 3- and 5-month-olds. In the first experiment, they found that, unlike the 6-months-olds in Bertin and Bhatt (2004), 3-month-old infants could not detect a thatcherized face following habituation to a normal face- this was true for both upright and inverted thatcherized faces. In the second study, they used a slightly younger age group than the Bertin and Bhatt study (5-month-olds) in order to replicate and extend their finding that 6-month-olds are sensitive to second-order relational information. Employing a more direct second-order relational change (moving the eyes out and the mouth down, similar to the Thompson et al. (2001) manipulations, instead of thatcherization) with a female face, they found evidence of second-order processing in infants: When habituated to a distorted second-order face and tested with the same face paired with a normal face, 5-montholds looked longer at the novel, more prototypical face. Three-month-olds, on the other hand, 
failed to discriminate. The authors concluded that the capacity to process second-order information develops sometime between 3 and 5 months of age.

In summary, the previous research suggests that, while it is debatable whether infants and young children are as adept at utilizing second-order relational information in identification as older children and adults, they do show sensitivity to this type of information at as young as five months of age.

\section{The Present Study}

The Bhatt et al. (2005) study suggested that infants as young as 5 months of age are sensitive to second-order relational information. However, there is uncertainty about this conclusion because in that study, the manipulation of second-order relational information was well outside of the normal range for female faces. In the current series of experiments, we felt that it was important to manipulate faces within the normal range of the population because some researchers (Mondloch et al., 2004, 2006) believe that manipulation of metric distances between features to the point that the distances are outside of the normal range may alter the face to be outside of prototypical 'facedness.' Therefore, in the current study, we took great care to ensure that the manipulation of the metric distances between the features of the face remained within the normal range of the population (Farkas, 1994) so that we could examine whether infants are sensitive to second-order changes within the normal range seen in "real-world" faces.

We also addressed another issue in this study that pertains to the nature of the stimuli used in face processing studies. Research suggests that photographs and line drawings are processed differently. Some studies indicate that line drawings of faces are not recognized as well as photographs of faces (Leder, 1996, 1999; Tversky \& Baratz., 1985)--shading and depth information are lost when line drawings depict faces. Other researchers suggest that the use of photographs provide so much detail that young children become confused by realistic photographs because it is difficult for them to keep in mind that they are seeing a representation of another object because the photograph is itself so compelling (DeLoache, Pierroutsakos, \& Utal, 2003). Felician et al. (2003) (see also Slaughter \& Heron, 2004) suggest that, because photographs are so complex, they require more perceptual and visual attention, making a photograph more difficult to process than a line drawing.

More relevant to the present study, Schwarzer \& Zauner (2003) found that 8-month-old infants processed features in the context of configural information in a photograph of a human 
face, but processed features independently of the configural information in a line drawing of a human face. Thus, the Schwarzer \& Zauner (2003) study indicated that line drawings of faces are processed differently than photographs.

In light of the research suggesting that line drawings and photographs are processed differently, we felt that it was important to examine infants' sensitivity to second-order relational information with stimuli that were as close to what they experience in the real-world as possible. Therefore, in the present series of studies, we examined infants' sensitivity to second-order relational information using photographs of female faces in which shading and depth information remained intact. Specifically, we conducted three different types of studies in order to explore 5and 7-month-olds' sensitivity to second-order relational information.

The first type of study involved a paired-comparison procedure [like the one used by Thompson et al. (2001)] in which infants were presented with a normal face paired with a face in which the second-order relationships in the face had been distorted. This type of study tested sensitivity based on "spontaneous" preference for one face over another and attempted to replicate the Thompson et al. study without altering featural information.

The second type of study involved a habituation-novelty preference procedure like the one used by Bhatt et al. (2005) in which infants were habituated to a face and tested with the familiar face paired with a face with novel second-order relational information. This methodology served as a finer tool for assessing discrimination, as the study allowed infants to sufficiently encode one face prior to being shown the alternate face, thus differing the amount of novelty between the faces.

The final method of studying sensitivity to second-order information was a relatively new procedure in our lab called the motion-enhanced discrimination procedure that capitalized on infants' attraction to movement. During the course of 16 trials, infants viewed a pair of faces: an unaltered face paired with same face in which the second-order relational information had been altered. In each trial, after a period of time in which the pair of faces remained stationary (2, 4, or 8 seconds), one face moved while the other remained stationary. A previous study using this technique to explore discrimination found that over the course of several trials, during tests in which both images remained immobile, infants began to look towards the image that had remained stationary during prior periods of movement (Bhatt, Hayden, Reed, Bertin, \& Joseph, 2006). In other words, infants exhibited discrimination by looking longer at the pattern that had 
not moved in previous trials, presumably because the other pattern (the one that moved) had become more familiar to the infants as a result of movement in previous trials attracting their attention and processing. In the Bhatt et al. (2006) study, this methodology proved to be more sensitive than a typical familiarization/novelty preference procedure. We adopted the motionenhanced discrimination procedure in the current study as an additional method for examining infants' sensitivity to second-order information.=

We used these three discrimination methods to examine whether 5- and 7-month-olds are sensitive to second-order information in faces within the normal range of variability seen in the "real world". 


\section{Chapter 2 \\ Experiment 1}

This study tested 7-month-olds' sensitivity to second-order information using the spontaneous-preference/paired-comparison procedure employed by Thompson et al. (2001), who found that infants preferred to look at a more prototypical face rather than a face in which the second-order information (and, unintentionally, the featural information) had been distorted. In the current experiment, we presented two faces that were identical except for a change in secondorder relational information. We expected that, if infants found one of these faces to be more prototypical than the other, they would prefer to look at this face over the other face.

\section{Method}

\section{Participants}

This study involved 16 7-month-olds (12 males, 4 females; mean age $=201.63$ days, $S D$ =9.52). These infants were recruited using local birth announcements. Parents received a participation certificate with their infant's name, a bib, and \$5 for transportation costs. Stimuli

A color photograph of a female Caucasian face was obtained from the Research Network on Early Experiences and Brain Development. ("Development of the MacBrain Face Stimulus Set was overseen by Nim Tottenham and supported by the John D. and Catherine T. MacArthur Foundation Research Network on Early Experience and Brain Development. Please contact Nim Tottenham at tott0006@tc.umn.edu for more information concerning the stimulus set.” We are unable to publish this photograph because we could not obtain permission to do so. However, for those who wish to view the face by accessing the MacBrain web site, this face is listed as face number 8.) Using Adobe Photoshop, this face was distorted in the same way the female face in the Bhatt et al. (2005) study was distorted: her eyes were moved out and her mouth was moved down. This alteration modified the second-order relationships in this female face-specifically, the metric distances between the eyes and between the nose and the mouth. We used the anthropomorphic averages determined by Farkas (1994) in manipulating the secondorder relationships in the face. The undistorted interocular distance for this face was approximately -3.44 SD from normal. Following manipulation of her eyes, the eyes were approximately -.85 SD from normal. The eyes were moved a total of $4.5 \mathrm{~mm}$ (a visual angle of .57 degrees). The undistorted nostril-to-top-lip measurement was approximately -.69 mm from 
normal. The manipulation resulted in her mouth being approximately .17 SD from normal. Her mouth was moved down $3 \mathrm{~mm}$ (a visual angle of .38 degrees). Both the original face and the distorted face had metric distances between the features that were within the normal range for adult female human faces (Farkas, 1994). According to Farkas' norms, the changes in the space between the eyes involved a 2.59 SD change, while the mouth displacement involved a .86 SD change. These changes were similar to the $2.60 \mathrm{SD}$ (eyes) and 1.06 SD (mouth) changes employed by Mondloch et al. (2002) to examine second-order processing in children. We nicknamed this face "Jenny" so as to avoid confusion in the following experiments in which we employed more than one face.

\section{Apparatus}

Infants were seated in a comfortable chair on the mother's lap approximately $45 \mathrm{~cm}$ in front of a $50 \mathrm{~cm}$ IBM computer monitor. This setup took place in a darkened chamber, which was located in the corner of a room. The darkened chamber restricted light from entering in order to focus the infants' attention to the screen, which was the only source of light in the chamber. A Sony CCD-FX430 camera, located on top of the computer monitor, recorded the session so that coding could take place in an alternate location off-line. The experimenter viewed the infant on a TV monitor attached to the camera. Sessions were recorded using a Sony DVD recorder. The TV, the DVD recorder, and the computer used to run the experiment were located outside of the testing chamber.

Procedure

There were a total of 2 spontaneous-preference trials for each infant. On each trial, the infant's attention was drawn to the center of the screen by the flashing of colorful shapes and by taps on the back of the computer meant to ensure that the infant would not have a side bias prior to the presentation of the test stimuli. Once the infant directed his/her gaze towards the center of the screen, the experimenter pushed a button to bring up the stimuli on the screen. A simultaneous pairing of two upright faces (normal, distorted) appeared on the screen and remained for 20 seconds. An interstimulus interval of approximately 2-3 seconds followed in which the infants once again viewed the attention-getter (flashing shapes). The same faces reappeared for another 20 seconds following the interstimulus interval. The left-right location of the faces on the first trial was counterbalanced across infants. The location of the faces was also switched from one trial to the next. 
A coder, blind to the infant's experimental condition, coded, off-line, the test trials at a speed 11 times slower than normal. A second coder coded $20 \%$ of the same infants so as to obtain reliability measurements. The Pearson correlation between the two observers' scores was .96. To assess discrimination, a ratio of total looking time to the normal face divided by the total looking time to both faces (normal and distorted) was calculated for each infant. The ratio was multiplied by 100 to get a percent value.

\section{Results}

As Table 1 indicates, infants failed to exhibit a preference during the test. Their preference score of $48.50 \%$ was not significantly different from the chance level of 50\%, [t(15)= $-.728, p>.1]$.

\section{Discussion}

Seven-month-olds failed to show a preference for one face over another in this procedure. This result suggests that the images were equally prototypical to these infants (or that the difference in prototypicality was not enough to ensure a spontaneous preference for one face over another). This failure to exhibit preference does not necessarily indicate a failure to discriminate because infants may have actually processed the differences between the two faces but may not have found one face to be more attractive than the other. Thus, in the following experiment, we used a discrimination procedure that involved familiarizing infants to one face and then examining whether they prefer a novel face that differed from the familiar face in terms of second-order information. 
Table 2.1, Mean Preference Score (percentage) for the Normal Face During the Spontaneous Preference Test Trials of Experiment 1

$N \quad M(S D) \quad t$ (vs. chance level of $50 \%)$

Normal Face Preference $\quad 16 \quad 48.50(8.22) \quad-.728^{@}$

${ }^{@} p>.10, \mathrm{n} . \mathrm{s}$.

Copyright @ Angela Hayden 2006 


\section{Chapter 3}

\section{Experiment 2}

Infants in Experiment 1 failed to show a preference between a normal female face and the same face with second-order relational alterations. We conducted the next experiment using a habituation procedure because Bhatt et al. (2005) had previously found discrimination of secondorder changes using a habituation procedure. In the current experiment, the normal and distorted female face that served as stimuli in the first experiment were again included. Infants were habituated to one of the two stimuli and tested for their preference between that pattern and the other. If infants can discriminate between the two patterns, then they should exhibit a preference for one of the patterns. In addition, in order to be able to generalize beyond one specific female face if infants showed evidence of discrimination, the second-order relational information of a second female face was manipulated and utilized in this procedure.

\section{Method}

\section{Participants}

This study involved 16 7-month-olds (7 males, 9 females; mean age $=194.06$ days, $S D=$ 8.92). Two infants were excluded due to side preference (defined as looking towards one side of the screen greater than or equal to $95 \%$ of the time during the test trials), and one infant was excluded for preferential looking (defined as looking towards one stimulus over another greater than or equal to $90 \%$ of the time during the test trials).

Stimuli

The photographs of the normal and distorted female face that were used in Experiment 1 (Jenny) were also used in Experiment 2. A second female Caucasian face served as an alternate stimulus (See Figure 3.1). The face of the woman in the photograph was removed from the background of the original photo and placed in Adobe Photoshop onto a white background. As in Experiment 1, the experimenter manipulated the face by moving the woman's eyes out and her mouth down. Again, we used the Farkas (1994) norms in order to manipulate the second-order relational information in the face within normal parameters for an adult female face. The undistorted interocular distance for this face was approximately -.30 SD from normal. Following manipulation of her eyes, the eyes were approximately 2.47 SD from normal. This woman's eyes were moved $4.5 \mathrm{~mm}$, or 2.77 SD (a total visual angle of .57 degrees). The undistorted nostril-totop-lip measurement was approximately $-.23 \mathrm{~mm}$ from normal. The manipulation resulted in her 
mouth being approximately .38 SD from normal. Her mouth was moved down 2 mm, or .61 SD (a total visual angle of .25 degrees). We nicknamed this woman’s face "Pam” so as to avoid confusion with the “Jenny” face.

Apparatus

The equipment and room setup was identical to that of Experiment 1.

Procedure

A habituation/novelty preference procedure was used for this study (e.g., Bhatt \& Bertin, 2001; Bhatt et al., 2005). Prior to the beginning of every trial, an attention-getter (a rapidly pulsating red-blue-green pattern) was presented in the center of the screen. The experimenter pressed a key to begin the trial when the infant looked towards the attention-getter. Following the key-press, two identical habituation patterns were presented, one on each side of the screen. These patterns remained on the screen until the infant looked away for 2 seconds or more or if 60 seconds had elapsed with the infant looking at the patterns. These trials continued until the infant's looking time average over three consecutive looking trials had decreased to $50 \%$ of the looking time average on the first three trials (or until a maximum of 20 habituation trials had been completed). Thus, both the length of the trials and the total number of trials were controlled by infant looking. Following habituation, infants were tested for their preference between the two different organization changes in two 10-second test trials. The initial left-right presentation of the stimulus pairs was counterbalanced across participants. The positions of the test stimuli were switched between test trials as well.

The face with which infants were habituated was counterbalanced between infants: half of the infants were habituated to the Jenny face, others were habituated to the Pam face. Within each face condition, half of the infants were habituated to the distorted face and half to the normal face. A coder, blind to the infant's experimental condition, coded, off-line, the test-trials at a speed 4 times slower than normal (and a separate coder coded 20\% of the same infants to obtain reliability measurements- the Pearson correlation between the two observers' scores was .94). As in Experiment 1, a ratio of total looking time to the novel face divided by the total looking time to both faces (novel and familiar) during the test was calculated for each infant. The ratio was multiplied by 100 to get a percent value. 


\section{Results}

Table 3.1 displays infant look durations for the habituation trials. A trial (first three, last three) X condition (normal face, distorted face) X face (Jenny, Pam) analysis of variance (ANOVA) performed on the look duration data during habituation indicated a trial main effect: $F(1,12)=14.94, p=.002$. No other main effect or interaction was significant in this analysis. Therefore, infants were habituated prior to the test trials and there were no differences in habituation to the Jenny versus Pam faces of either type.

As described above, preference during the test trials was assessed by computing a percentage score that indicated preference for the novel stimulus. Preference scores are displayed in Table 3.1. The overall average score of $49.37 \%$ was not significantly different from the chance level of $50 \%, t(15)=-.27, \mathrm{p}>.1$, indicating a failure to discriminate between the two faces.

This result seems to suggest that 7-month-olds had failed to discriminate between faces that differed in terms of second-order information within the typical range. However further analyses suggested a more complex scenario. This followed from the conducting of a condition (normal, distorted) X face (Jenny, Pam) ANOVA, which revealed that, although there was not a main effect for face or an interaction between the two variables, there was a main effect of condition $(F(1,12)=5.79, p=.03)$. This main effect indicated a significant difference in the performance of those habituated to the normal pattern versus those habituated to the distorted pattern. As Table 2 indicates, infants who were habituated to the normal face exhibited a 44.24\% novelty preference, whereas those habituated to the face in which the second-order relational information had been altered exhibited a 54.51\% novelty preference. In other words, infants habituated to the normal face exhibited a familiarity preference, whereas those habituated to the distorted face tended to exhibit a novelty preference. Indeed, all infants that had been habituated to the normal face showed a familiarity preference, and their mean score of $44.24 \%$ was significantly different from the chance level of $50 \%, t(7)=4.48, p<.01$. Thus, infants habituated to the normal face exhibited evidence suggestive of discrimination, although this was in the form of a familiarity preference. The average score of those habituated to the distorted face (54.51\%) was not different from the chance level of 50\%. Thus, infants habituated to the distorted face failed to exhibit evidence of discrimination. 
Clearly, these findings were unexpected. However, they suggest that, at least under some circumstances, infants may be sensitive to second-order relational information.

\section{Discussion}

Seven-month-old infants failed to discriminate between the altered and distorted faces according to the overall average scores. However, further analyses revealed that, in the case of those habituated to the normal face, no matter which face the infants were habituated to (Jenny or Pam), the preference for the familiar face was unanimous: every infant in that condition preferred the familiar face over the novel face. In fact, the preference for the familiar face was significantly different from chance levels with only eight infants. This differential encoding of the normal and distorted faces could be the result of preferences brought out by the procedure. In Experiment 1, infants presented with two different but relatively similar faces may not have shown a preference for one over the other because both stimuli were novel and neither was so non-prototypical as to direct the infants' looking toward the other. Repeated exposure to a single face in Experiment 2, however, may have resulted in a different encoding level for normal versus distorted faces as infants became habituated and the faces lost their novelty. Alternatively, the habituation procedure may have brought out preferences during the test trials. Perhaps during Experiment 1, the initial arousal from seeing faces prevented infants from preferring one face over another. This excitement may have dissipated during the course of the habituation trials in Experiment 2, and infants could then exhibit preferences for one face over another during the test trials.

While the strong familiarity preference in one condition of the current experiment was not strong enough evidence to conclude that infants were sensitive to second-order relational information in general, we felt that this was enough evidence to pursue other methodologies that would perhaps be more sensitive to infant discrimination. 
Table 3.1 Mean Fixation Times (seconds) During the Habituation Trials and Preference Scores (percentages) for the Novel Face During the Test Trials of Experiment 2

\begin{tabular}{|c|c|c|c|c|c|}
\hline \multirow[b]{3}{*}{ Habituation Face } & \multicolumn{3}{|c|}{ Habituation Trials } & \multicolumn{2}{|c|}{ Test Trials } \\
\hline & & $\begin{array}{c}\text { First } \\
\text { three trials }\end{array}$ & $\begin{array}{c}\text { Last } \\
\text { three trials }\end{array}$ & $\begin{array}{l}\text { Preferenc } \\
\text { the novel }\end{array}$ & $\begin{array}{l}\text { e for } \\
\text { face }\end{array}$ \\
\hline & $N$ & $M \quad(S D)$ & $M \quad(S D)$ & $M \quad(S D)$ & $t$ \\
\hline Normal Face & 8 & 31.82 (18.57) & $12.44(6.93)$ & $44.25(4.48)$ & $-3.64^{*}$ \\
\hline Distorted Face & 8 & 25.19 (17.68) & 10.21 (2.99) & $54.51(18.02)$ & 1.24 \\
\hline Combined & 16 & $28.50(17.85)$ & $11.33(5.28)$ & 49.37 (9.30) & -.271 \\
\hline
\end{tabular}

$* p<.01$, two-tailed. 

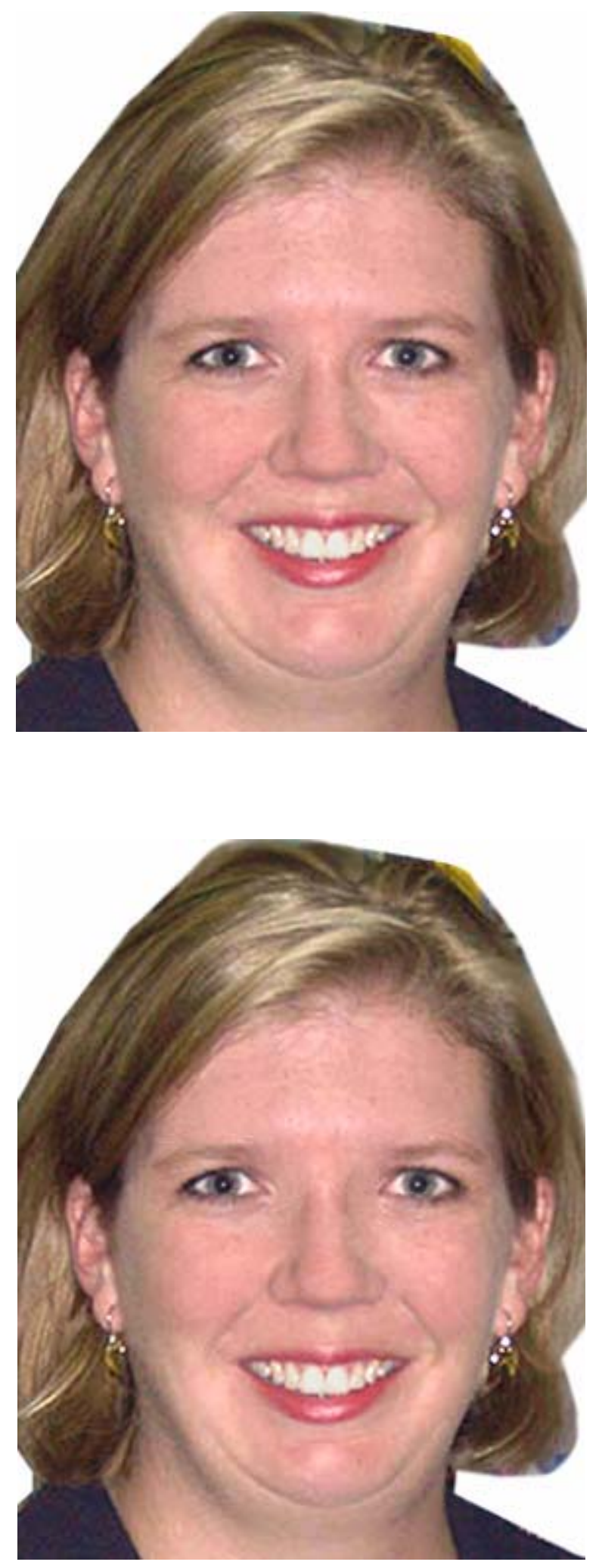

Figure 3.1. Normal Pam face on the top and the Pam distorted face on the bottom. 


\section{Chapter 4}

\section{Experiment 3}

In a recent study, Bhatt et al., 2006 successfully employed a new technique to assess discrimination by infants. This technique used movement as a method of alerting infants to differences between stimuli. The technique was originally conceived as an expectancy procedure that was based on the studies conducted by Haith and his colleagues (e.g., Haith \& McCarty, 1990) in which infants learned to predict, based on location, the appearance of an object or event. Infants in these studies exhibited expectancy behavior by looking toward the location of an event that would take place in that particular location in the future- prior to the actual occurrence of the event. We altered this procedure to make the content of the stimuli, rather than the location of an event, predictive of future change. In our procedure, infants concurrently viewed two different stimuli on each trial. At the beginning of the trial, these stimuli remained stationary for a fixed period of time. Then, one of the stimuli moved. This same stimulus moved in all of the trials. Bhatt et al. (2006) found that, during the stationary periods prior to movement, infants looked longer towards the stimulus that remained immobile than towards the stimulus that had moved during the periods of movement in prior trials. This was an unexpected occurrence, as we anticipated that, as in the Haith study, infants would look towards the object that would move in the future rather than the object that remained stationary throughout the procedure. However, we replicated the outcome of this procedure with the same stimuli and have since replicated this finding with several studies, bolstering our confidence in this procedure.

We reasoned that the greater looking toward the nonmoving pattern is a novelty-based response induced by the fact that infants in previous trials had more deeply encoded the moving stimulus. This account is supported by the fact that infants in the Bhatt et al. (2006) study were attracted by the movement and looked towards the moving stimulus when it moved. We reasoned that, while this image was moving, infants were also encoding its content. Therefore, when shown two stationary images, one which they had just seen move (and had been encoding) and one which they had not (and thus had not been encoding), the image that remained stationary throughout the procedure is relatively more novel to them. Therefore, we believe that this procedure closely resembles a habituation procedure, in which infants become habituated to the moving stimulus and look more toward the nonmoving pattern in test trials in which both 
patterns are stationary. Therefore, we called this procedure the movement-enhanced discrimination procedure.

Bhatt et al. (2006) found that the movement-enhanced discrimination procedure was more sensitive than a traditional familiarization-novelty preference test procedure: Infants tested using the former procedure exhibited discrimination with stimuli that they had failed to discriminate when the latter procedure was used. The movement-enhanced discrimination procedure may be more sensitive than novelty preference tests because the differences between stimuli are underscored by the movement of one and not the other and there are several testing opportunities (eight 8-second test trials as opposed to the two short 10-second test trials in the habituation procedure). Also, the procedure itself is more engaging to the infants.

In the present study, infants saw a normal face paired with a face in which the secondorder information had been altered. Following the template of the previous study (Bhatt et al., 2006), infants were exposed to trials in which the faces remained stationary for a period of time, followed by the movement of one of the faces. Based on the results of the previous study, we expected that, during the stationary test periods, infants would look more towards the image that remained immobile if they could discriminate between the two images.

\section{Method}

\section{Participants}

This study involved 24 7-month-olds (13 males, 11 females; mean age $=199.75$ days, $S D$ $=8.39$ ). These infants were recruited in the same manner as those in the studies above. Two infants were excluded due to fussiness.

Stimuli

The stimuli were identical to those used in experiment 2 . Thus, both the Jenny face as well as the Pam face were used in this study.

Apparatus

The apparatus was identical to that used in experiments 1 and 2.

Procedure

Infants were tested using a 16-trial movement-enhanced discrimination procedure (Bhatt et al., 2006). At the beginning of each trial, the experimenter drew the infant's attention to the center of the screen. When the infant's attention was engaged, the experimenter pushed a button to display stimuli: an unaltered face appeared on one side of the screen and a face with second- 
order alterations appeared on the opposite side. Half of the infants, randomly determined, saw the Jenny face and the corresponding second-order altered face, while the other half saw the Pam face and the corresponding altered face. A period of 2, 4, or $8 \mathrm{~s}$ followed in which the faces remained stationary on the screen. The length of the stationary period was quasi-randomly determined. Each four-trial run (i.e., trials 1-4, 5-8, 9-12, and 13-16) was considered a 'block.' Within each block, there was one 2-s trial, one 4-s trial, and two 8-s trials. The two 8-s stationary periods within each block were considered the 'test trials' for this procedure because they provided enough time for infants to locate and fixate upon the image that they preferred. The order of presentation of the 2-s, 4-s, and 8-s trials within each block was randomly determined. The length of the trial (i.e., whether the trial was 2, 4, or 8 seconds) was randomly determined so as not to cue infants to when the image would move. This would, we reasoned, prevent a fixed-interval scallop in looking behavior in which infants would look a greater amount towards the latter part of the stationary period because they would be able to predict when the image was going to move based upon the length of the stationary period. Instead, we were anticipating a variable-interval-type looking behavior in which infants consistently looked towards the screen in anticipation of movement because they were unsure of exactly how long the images would remain stationary. Following the stationary period, a 6-s period of musicaccompanied action followed. For half of the infants, randomly determined, the altered face moved; for the other half, the unaltered face moved. The faces moved in 3 different ways or shrank and expanded on the screen. Two different orders of the 16 trials were used in this study. Each order was quasi-randomly determined such that the moving face appeared in opposite locations (left/right) during the two 8-second test trials in each block, there were equal numbers of left and right movement trials over the course of the 16 trials, and there were no more than three consecutive trials in which the moving face was in a particular location (left or right). The 8-s test trials were treated similarly to the test trials in Experiments 1 and 2. That is, infants' preferential looking toward the non-moving versus moving patterns during these 8-s stationary periods were used to assess discrimination. As in prior experiments, coding of discrimination performance during these test trials was conducted off-line. The DVD player was slowed to 25\% of the normal speed during coding. A different coder coded $20 \%$ of the infants in order to obtain a reliability measure. The Pearson correlation between the two observers' scores was .98. 


\section{Results}

A ratio of total looking time to the non-moving face divided by the total looking time to both faces (normal and altered) was calculated from the 8-s test trials to get an overall preference score for the moving versus the non-moving stimuli. The ratio was multiplied by one-hundred to get a percent value. A face (Jenny, Pam) X condition (distorted, normal) ANOVA suggested that there were no main or interaction effects. Therefore, performance was not affected by whether the infants were tested on the Jenny or Pam faces, nor was performance a function of whether the normal or the distorted face was the moving pattern. We therefore collapsed across conditions in the following analyses.

As Table 4.1 illustrates, a $t$-test examining the overall looking time when compared to chance revealed that 7-month-olds looked significantly longer towards the image that remained stationary throughout the experiment $(t(23)=-3.38, p<.01)$. In addition, 18 out of the 24 infants in this experiment showed this preference. A binomial test confirmed that this outcome was highly unlikely to be due to chance $(p=.02)$. Thus, 7 -month-olds clearly discriminated between the unaltered and second-order distorted faces in this experiment.

\section{Discussion}

Seven-month-old infants showed clear evidence of sensitivity to second-order information in this experiment. They exhibited discrimination between two faces that differed in terms of second-order relational information. Moreover, unlike in Experiment 2, in which evidence of second-order processing was confined to infants who were habituated to the normal faces only, here infants exhibited discrimination in all conditions-with both Jenny and Pam faces and independent of whether the altered or the unaltered pattern was the moving pattern. Thus, the movement-enhanced discrimination procedure used in this study yielded clear evidence of second-order processing by 7-month-olds. In the next experiment, we sought to replicate the results of the current experiment and to extend them to a younger age group, namely 5-montholds. 
Table 4.1 Mean (and Standard Deviation) of Percent Preference to the Non-Moving Upright Face for 7-Month-Olds in Experiment 3

$N \quad \mathrm{M}(S D) \quad t$ (vs. chance level of $50 \%)$

Non-Moving Face Preference $24 \quad 53.70$ (5.37) 3.38*

$* p<.005$, two-tailed 


\section{Chapter 5}

\section{Experiment 4}

In the previous experiment, we found that 7-month-olds are sensitive to changes in second-order relational information that are within the normal range for human faces using the movement-enhanced discrimination procedure. In the current experiment, we used the same procedure to examine whether this sensitivity extended to 5-month-old infants. Five-month-olds discriminated second-order information changes in Bhatt et al. (2005) when habituated to line drawings of faces in which the relational information had been altered beyond normal range. In the present study, we explored 5-month-olds' sensitivity to second-order information in faces that were within the normal range.

We also explored the inversion effect in the current experiment. According to some researchers (e.g., Diamond \& Carey, 1977; Freire et al., 2000; Goldstein \& Chance, 1980; Itier \& Taylor, 2004), inverting a face disrupts relational processing, forcing people to rely mainly on featural information for identification. The changes in spacing among features that we made to the faces used in the current experiments are typically considered to be second-order relational changes (Mondloch et al., 2002, 2004, 2006; Thompson et al. 2001) and were the same as those used in other studies that have examined second-order relational information processing in children and adults (e.g., Mondloch et al. 2002, 2006; Murray, Yong, \& Rhodes, 2000; Searcy \& Bartlett, 1996). Therefore, if infants discriminate in the upright condition but not in the inverted condition, then it would suggest that 5-month-olds are sensitive to second-order relational information and process this information in a manner similar to adults.

Another motivation for the examination of the inversion effect in the current experiment was the suggestion by Diamond and Carey $(1986,1994)$ that the inversion effect associated with second-order relational information is an indication of face-processing expertise. Diamond and Carey (1986) demonstrated that humans show inversion effects when processing faces but not when they are processing landscapes. In a separate experiment, they presented results suggesting that dog experts show an inversion effect when discriminating between dogs of breeds with which they have expertise, whereas people who are not dog experts fail to show the inversion effect. The outcomes of both of these experiments suggest that only people that have had enough exposure to an object category to gain expertise with it will show inversion effects, meaning that they can more efficiently identify the object in its canonical orientation than in an 
inverted orientation. Therefore, the presence of an inversion effect would indicate that infants at 5 months of age have had the necessary exposure to faces to have gained at least rudimentary levels of face processing expertise.

\section{Method}

\section{Participants}

Participants were 32 5-month-olds infants (19 females, 13 males; mean age $=152.41$ days; $S D=5.78$ days). They were recruited in the same manner as the infants in the first three studies. One infant was excluded due to equipment failure.

Stimuli

The faces used in this study were identical to those used in Experiment 3 (Jenny and Pam). In addition, inverted versions of the Jenny and Pam faces were used to explore the inversion effect.

\section{Apparatus}

The apparatus was identical to that used in experiments 1 - 3.

\section{Procedure}

As in Experiment 3, infants participated in the movement-enhanced discrimination procedure. The variations that were used in Experiment 3 (two different movement patterns, the Jenny and Pam faces, either the distorted or the normal face moving) were also used in this experiment.

Recall that in Experiment 3, infants were exposed only to upright faces. In the current experiment, one group of infants was tested on upright faces, while a separate group of infants was tested on the same faces presented inverted throughout the procedure (i.e., on all test and non-test trials). In all other respects, infants in the inverted group were treated in the same manner as infants in the upright condition. We reasoned that if infants fail to discriminate in the inverted condition under the same conditions in which they discriminate upright faces, then it would suggest that infants employed second-order relational information in discrimination and that they are beginning to exhibit evidence of expertise in face processing.

\section{Results}

Again, we conducted analyses based upon overall looking percentages over the course of the 8 test trials. An orientation (upright, inverted) X face (Jenny, Pam) ANOVA indicated a main effect of orientation $(F(1,32)=4.32, p<.05)$. There were no other main effects or 
interactions. Therefore, only the orientation factor affected infant looking, indicating that the preference score was significantly greater in the upright than in the inverted condition.

As Table 5.1 illustrates, individual $t$-tests revealed that infants discriminated between the two faces in the upright condition $(t(15)=-2.22, p<.05)$, but not in the inverted condition $(t(15)=.704, p>.1)$. Eleven of the 16 infants in the upright condition exhibited a familiarity

preference. While a binomial test revealed that this result was not significantly different from chance $(p=.21)$, as described above, a parametric $t$-test clearly indicated discrimination by infants in the upright condition. Only 7 of the 16 infants in the inverted condition exhibited a familiarity preference. A binomial test suggested that this was at chance level $(p=.80)$. Like the 7-month-olds in Experiment 3, 5-month-olds in the upright condition of the present experiment exhibited discrimination of second-order information changes by looking towards the face that remained stationary throughout the study. However, infants failed to discriminate the same changes when the faces were presented upside-down, thereby exhibiting an inversion effect.

\section{Discussion}

Five-month-olds in the current experiment exhibited clear evidence of sensitivity to second-order relational information with the movement-enhanced discrimination procedure. This result replicates and extends the results obtained with 7-month-olds in Experiment 3 to 5month-olds. Moreover, infants in the current experiment showed evidence of an inversion effect, suggesting that, like adults, infants process faces more efficiently when the faces are in their canonical orientation. Moreover, the inversion effect also suggests that infants as young as 5 months of age have the necessary tools to become experts at face processing.

In the next study, we tested adults in a same-different reaction task in order to ensure that the second-order relational information that we manipulated in the stimuli that the infants viewed (the Jenny and Pam faces) was within normal limits. 
Table 5.1 Mean (and Standard Deviation) of Percent Preference to the Non-Moving face (in both the upright and the inverted conditions) for 5-Month-Olds in Experiment 4

\begin{tabular}{cccc} 
& $N$ & $\mathrm{M}(S D)$ & $t(\mathrm{vs}$. chance level of 50\%) \\
\hline Upright Faces & 16 & $54.46(8.02)$ & $2.22^{*}$ \\
Inverted Faces & 16 & $48.77(7.01)$ & -.704 \\
\hline
\end{tabular}

$* p<.05$, two-tailed 


\section{Chapter 6}

\section{Experiment 5}

Recall that one of the key issues addressed by the current research is the question of whether infants are sensitive to second-order changes that are within the normal range of variability in human populations. To this end, when we manipulated the faces used in the current experiments, we were very careful to ensure that the changes were within the normal range for faces of adult females as per the Farkas (1994) norms. Moreover, the spacing changes that were examined in the current experiments were similar to (and in some cases smaller than) the changes used by Mondloch et al. (2002, 2004) to study sensitivity to spacing changes in childhood.

Further evidence that the stimulus manipulations in the current experiments were within the normal range also comes from the fact that infants exhibited an inversion effect in Experiment 4. Mondloch et al. (2002, 2006) suggested that manipulating relational information outside of normal range fails to result in an inversion effect for adults. The fact that infants exhibited an inversion effect thus suggests that the stimulus manipulations in Experiments 1-4 were capturing relational information processing of the sort employed in discriminating between faces in a typical human population.

In the current experiment, we sought to obtain convergent evidence of the normality of the second-order changes by examining whether adults also exhibit an inversion effect, i.e., whether adults' discrimination performance on the faces used in Experiments 1-4 is better with upright than with inverted faces. If the spacing changes in these stimuli are outside of normal range, adults should not show an inversion effect. If, on the other hand, adults exhibit an inversion effect on the stimuli used in the current study, then it would be evidence that the spacing manipulations studied in the current experiments were within the normal range of variability in humans. Moreover, testing adults on the same stimuli that were used with infants also allowed us to examine whether there is some commonality of performance between adults and infants.

\section{Method}

\section{Participants}

Participants were 10 adults (6 females, 4 males; mean age $=29$ years, $S D=5.66)$. They were recruited via flyers, newspaper advertisements, and word-of-mouth. Some participants 
were compensated and others were not. This study was a part of 2 larger studies (an fMRI study and a behavioral study) and, therefore, compensation was determined by the larger study that participants took part in. Participants in the fMRI study were compensated \$25/hour. Participants in the behavioral study were compensated $\$ 15 /$ hour or through class credit. Stimuli

Participants participated in a familiarization session as well as a practice session prior to test sessions. The stimuli used in both the familiarization and practice sessions included grayscale depictions of computer-generated female faces. Using Faces 4.0 software, with which one can easily manipulate feature locations, the eyes on both faces were moved outwards and the mouths were moved downwards (similar to the manipulation used in the present study). Following the practice session, adults participated in the test session. They were tested on the Jenny and Pam normal and distorted faces that were used in Experiments 2-4 with infants. Procedure

\section{Familiarization Session}

This session was used in order to familiarize participants with the type of stimuli that they would be viewing. Adults were first comfortably seated in front of a computer screen. They then saw a screen with a typed sentence indicating that they would see some sample faces. This was followed by a sentence instructing the participants to look at the faces that would be presented on the monitor and to confirm for themselves that the faces were different. These instructions were followed by the presentation of a normal face paired with a face in which the second-order relational information was manipulated (described above). After the confirmation by the participant that the faces were different, a different face and its distorted counterpart appeared. Following these two upright face trials, participants viewed a screen with instructions asking them to confirm that two inverted faces were different. At the end of these trials, participants transitioned into a practice session in which they went through a series of trials that allowed them to practice the procedure they would have to follow during the test trials.

Practice Session

Participants were shown two faces, one located in the top half of the screen and the other located in the lower half. These faces were identical to those used during the familiarization phase (as described above). A single face (with and without second-order relational distortions) was shown during each block of 8 trials. On each trial, adults were shown two of the same face 
on the screen or a normal face in one position and the corresponding distorted face (eyes moved out, mouth moved down) in the other position. On half of the trials, the faces were inverted. The inverted faces followed the same pattern as the upright faces (i.e., at times the same face was presented on the screen, and at other times different faces were presented on the screen). The participant's task was to press buttons to indicate whether the two faces were the same (press the 'yes' button with their index finger) or different (press the 'no' button with their middle finger). The faces remained on the screen for $3000 \mathrm{~ms}$, regardless of whether the participant made their choice. There was a 1000 ms rest period between each trial. Feedback was not given at the end of the trial.

Participants saw every combination (inverted or upright, same or different) of a face (both normal and distorted versions of this face) during the course of one block. They saw two blocks of each face, for a total of 4 blocks, or 32 trials.

\section{Test Session}

The test session followed the practice session and was similar to it. The stimuli that the adults were tested on were identical to those used in Experiments 2-4 with infants. Stimulus presentations, trial durations, and the response requirements were the same as in the practice session, and, again, no feedback was provided to the subjects at the end of trials. Blocks

Participants were tested over 10 blocks, each containing 16 trials. One face (either Jenny or Pam) and its corresponding distorted version were presented in each block. Both inverted and upright faces were shown throughout the block in an attempt to prevent strategy formation for one orientation. The two faces in each trial had the same orientation (upright or inverted). Within these blocks, every possible trial type was presented: faces could be the same or different (and, if different, the normal face or the distorted face could be on the top or the bottom), or they could be inverted or upright. Participants saw each trial type once before the cycle was repeated. Half of the participants were tested on the Jenny face during the first 5 blocks, followed by the Pam face in the next 5 blocks. The other participants were tested in the reverse order. The normal face and the distorted face appeared equally often on the top and the bottom across trials, and there were an equal number of trials in which the participant saw an upright face or an inverted face. Participants saw each stimulus combination once during the course of each block. 
Following each set of 5 blocks, participants were allowed to rest if they wished- most chose to continue.

Results

Errors

Figure 2 depicts the errors made by the participants. A face (Jenny, Pam) X orientation (upright, inverted) X response ('same' response, 'different' response) ANOVA failed to reveal significant main effects of face or response. However, the main effect of orientation was significant $(F(1,9)=9.76 ; p<.02)$. This main effect suggests that participants made more errors when faces were inverted than when they were upright. However, this was qualified by a significant orientation X face interaction $(F(1,9)=4.50 ; p<.02)$. Further exploration of simple effects revealed that participants exhibited an inversion effect with the Jenny face $(F(1,9)=$ 14.78; $p<.01)$, but failed to exhibit an inversion effect with the Pam face $(F(1,9)=.027 ; p>1)$. Thus, participants exhibited clear evidence of an inversion effect in terms of errors with the Jenny face but not with the Pam face.

Reaction Time

Figure 4 depicts the reaction time data. As is customary in this type of research (e.g., Wilson et al., 1980), mean reaction times were log transformed to induce normal distributions before being analyzed. As is also common in this research, the reaction time analysis only included data from trials in which participants were correct. A face (Jenny, Pam) X orientation (upright, inverted) X response ('same' response, 'different' response) ANOVA failed to reveal a main effect of face or response. Participants exhibited a marginal inversion effect $(F(1,9)=$ $4.50 ; p=.06)$. There was no evidence of an orientation $\mathrm{X}$ face interaction $(F(1,9)=2.20 ; p=$ .17), suggesting that participants exhibited an inversion effect on both faces. Thus, unlike in the case of the error measure, performance in terms of reaction time indicated inversion effects (that were at least marginally significant) in the case of both Jenny and Pam faces.

\section{Discussion}

Adults in this study exhibited a marginal inversion effect with both faces according to the analysis of reaction time data. That is, adults were quicker to process differences or lack thereof in spacing among features when faces were presented upright than when they were inverted. This provides evidence convergent with the results of Experiments 2-4, which indicate that the manipulation of the second-order relational information was within normal range for both faces. 
Adults exhibited an inversion effect with the Jenny face based on the error data but not on the Pam face. However, the Farkas (1994) norms as well as infant discrimination in Experiment 4 suggest that the second-order information in the altered Pam face was manipulated within normal limits. One explanation for this divergent result with the two faces may be differences in strategy that participants used when processing these two faces.

An examination of the individual data for each participant in the adult study suggests that these participants tended to show a lack of an inversion effect only if they were tested on the Pam face first (see Figure 6.1). If they were tested on the Jenny face first, however, they had a tendency to show an inversion effect also on the Pam face. This supports the idea that participants may be using different strategies in discriminating these two faces. In any case, none of the experiments in which infants were tested revealed any difference in performance between the Jenny and Pam faces. Thus, infants seemed to use the same strategy for discrimination with both the Jenny and Pam faces. The Farkas (1994) measurements and the inversion effects obtained with adults in terms of the reaction time measure indicate that both faces are within normal range for the human population. This indicates that infants as young as 5 months of age are capable of discriminating second-order changes within the normal range of human variability. 


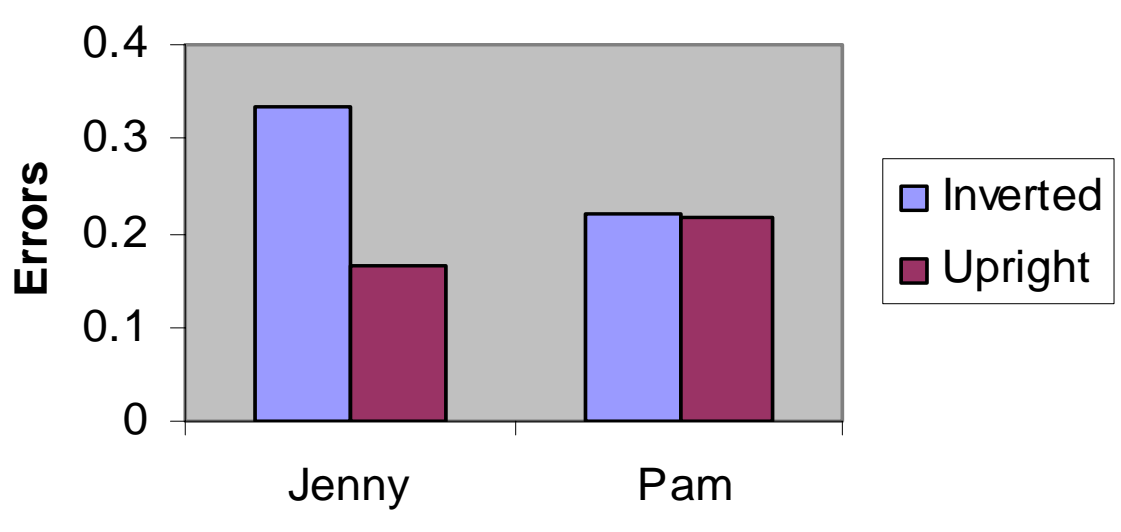

Face Condition

Figure 6.1. Mean error rate for the Jenny and Pam faces for upright and inverted trials. 

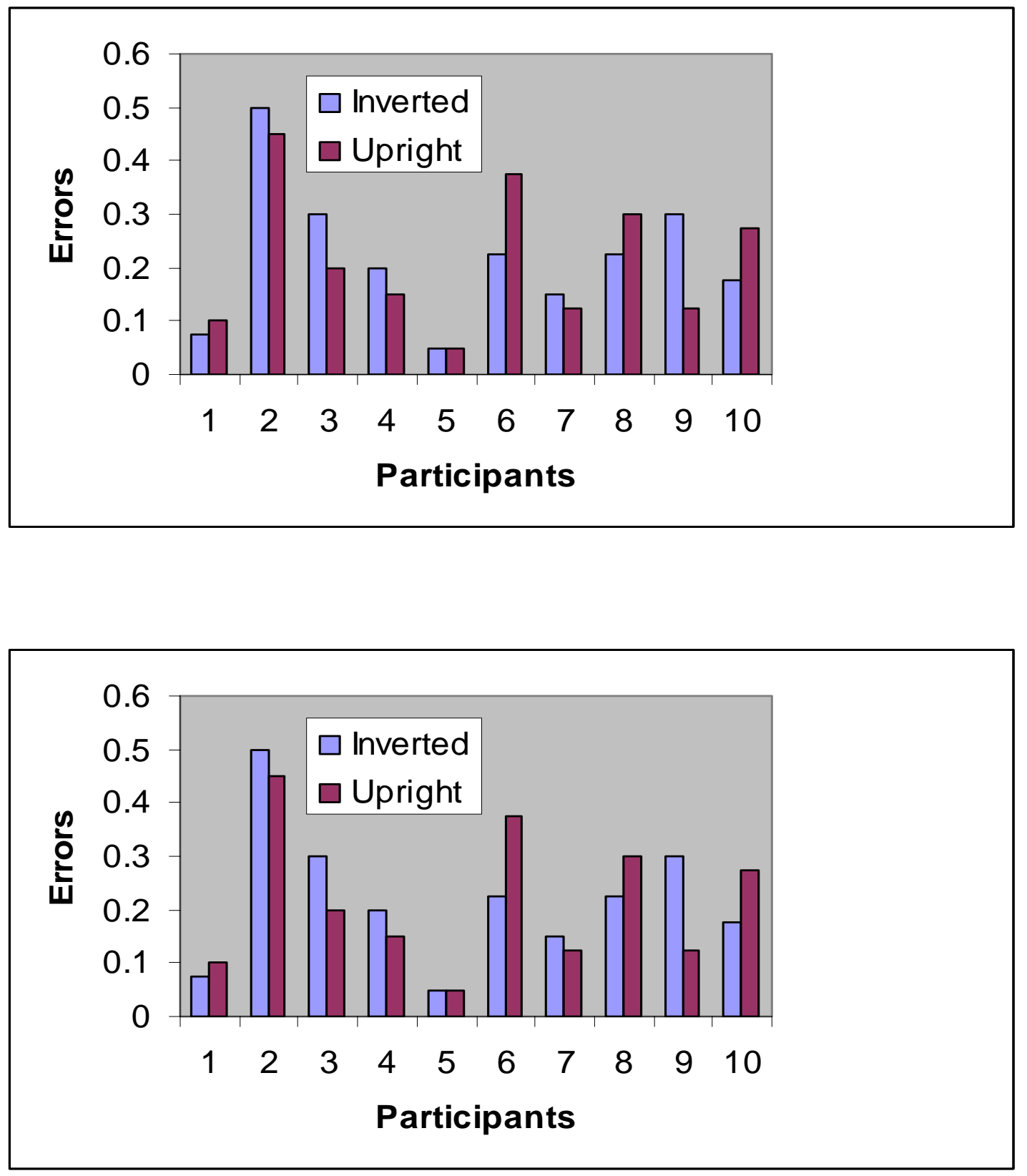

Figure 6.2. Individual error data for adults. The top graph shows errors made in response to the Jenny face. The bottom graph shows errors made in response to the Pam face. Participants 1 through 5 saw the Jenny face initially, followed by the Pam face. Participants 6 through 10 saw the Pam face initially, followed by the Jenny face. 


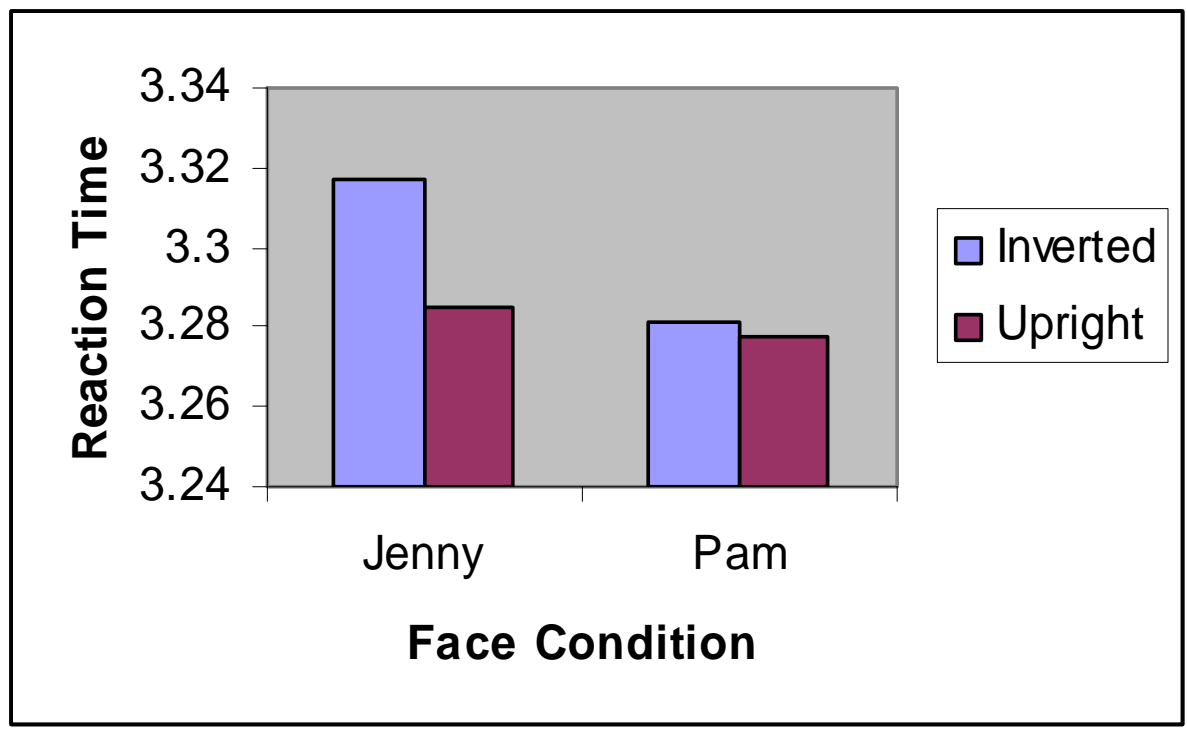

Figure 6.3. Mean reaction time (log transformed) to the Jenny and Pam faces for upright and inverted trials.

Copyright (C) Angela Hayden 2006 


\section{Chapter 7}

\section{General Discussion}

The results of this study indicate that both 5- and 7- month-olds are sensitive to changes in second-order relational information in faces that are within the normal range of human variability (see Figure 7.1 for an overview of all experiments). The results also suggest that infants as young as 5 months of age exhibit an inversion effect, thereby demonstrating a hallmark of facial second-order information processing by adults. To our knowledge, this is the first study to suggest that a critical mechanism of expert face processing, namely the processing of secondorder information in typical faces, is present within the first 5 months of life.

\section{Evidence of Second-Order Relational Manipulation Within Normal Range}

Comparison to Other Studies and Farkas Measurements

Bhatt et al. (2005) found that 5-month-olds are sensitive to second-order relational information. However, the stimuli used in Bhatt et al. were line drawings of faces in which the spacing between features was manipulated to a degree that was arguably out of normal human range. Some researchers (Mondloch et al., 2004, 2006) suggest that, when relational information is manipulated in such a way that it is beyond the bounds of human possibility, the information is no longer second-order information. Instead, they claim that these faces failed to conform to the normal human face structure because the features are located outside the bounds of typical human faces. In the current study, in contrast, we ensured that the relational information that was manipulated in the faces shown to both infants and adults was within the normal human range. The degree of manipulation of the relational information in our Jenny and Pam faces was similar in magnitude to that used in studies with children (e.g., Mondloch et al., 2004).

Moreover, the stimuli used in the current studies had characteristics that were within the normal range according to the anthropometric norms of Farkas (1994).

The Inversion Effect

Another piece of evidence for the normal nature of the second-order changes in the current experiments comes from the fact that inversion effects were exhibited by both infants and adults. This inversion effect meets the criterion for second-order processing that has been adopted by at least some researchers (e.g., Maurer et al., 2002; Mondloch et al., 2002). According to this criterion, the presence of the inversion effect suggests that infants as young as 5 months are encoding the information in faces in a fashion that is similar to adults. This means 
that both infants and adults encode and more readily recognize a face in its canonical orientation.

\section{Controversy Surrounding the Inversion Effect}

There is much debate in the literature as to the timeline of the initial emergence of the inversion effect in childhood. Separate studies have shown that 4-year-olds (Pellicano, et al., 2006), 6-year-olds (Brace et al., 2001), and 7- year-olds (Gilchrist \& McKone, 2003) exhibit an inversion effect when only the second-order information in a face is manipulated. Brace et al. (2001), however, found that 2- and 4- year-olds responded more quickly to inverted faces than upright faces, suggesting that neither age group showed an inversion effect. They did, however, find that 6-year-olds exhibited an inversion effect with the same task as they used with 2- and 4year olds. The varied results of these studies therefore suggest a lack of consensus among researchers as to when the inversion effect emerges. Itier \& Taylor (2004), who found that 8year-olds exhibited inversion effects similar to adults, suggested that inconsistencies in the outcomes of studies exploring the development of the inversion effect may be due to differences in measures and/or methodology. Pellicano et al. (2006), who found that not only were 4- and 5year-olds showing the inversion effect, but that they were exhibiting an inversion effect of the same strength as adults, concurred with Itier \& Taylor that inconsistencies in measures and/or methodology may be contributing to different study outcomes. They also suggested that the emergence and developmental strengthening of the inversion effect that studies had found may be due at least in part to an increase in general processing capacities rather than a specific increase in second-order relational processing associated with face recognition. According to Pellicano et al. (2006), children may have failed to show an inversion effect in some studies due to the difficult tasks employed in those studies.

The lack of consistency in methodology and the varied conclusions that different researchers have arrived at concerning the development of second-order information processing in childhood make it difficult to draw comparisons between prior developmental studies and the present study. In addition, the participants in the current study were much younger than those in most other studies that have examined the inversion effect. It is possible (albeit unlikely) that, due to the complexity of development, infants exhibit an inversion effect at 5 months but fail to exhibit this effect for a period of time prior to regaining it in early childhood. In order to arrive at a concrete explanation for the diverging results between infants and children regarding the 
inversion effect, further studies need to be conducted that employ standardized methodology to directly compare infants' and children’s performance

\section{Relationship of the Present Study to Previous Infant and Adult Studies}

Both Rose et al. (2002) and Thompson et al. (2001) showed 7-month-old infants photographs of real faces in which the second-order relational information was manipulated within normal human range. In both studies, infants exhibited sensitivity to the manipulated information. However, as noted above, in both studies there are questions about the nature of the stimulus manipulations, specifically concerning whether the changes were solely based on second-order relations. The results of the present study were consistent with those of Rose et al. and Thompson et al., but employed different procedures and a specific manipulation of secondorder relational information. Moreover, the present study found evidence of sensitivity to second-order relational information at 5 months of age.

While the current study suggests that infants as young as 5 months of age are sensitive to second-order information, Mondloch et al. (2006) concluded that even 4-year-olds lack sensitivity to second-order relational information. The divergence in results between Mondloch et al. and the current study [as well as Bhatt et al. (2005), Rose et al. (2002), and Thompson et al. (2002)] is most likely due to differences in complexity and memory load inherent in the tasks used in these studies. Studies with infants that have examined sensitivity to second-order relational information have been simple and direct. These studies strove to minimize memory demands and also attempted to simplify images so there is as little encoding of unnecessary information as possible. In contrast, children in Mondloch et al. were asked to remember (with the aid of reminders) two different children's faces for a span of two weeks. They were then asked to distinguish between the faces that they had seen (or their own face) and faces in which either the featural or the second-order information had been manipulated (or their own face with these manipulations). Mondloch et al.'s use of two different faces during familiarization (three, including participants' own face, during testing) required that these children encode twice the amount of featural and second-order relational information as participants in the present study. The memory-load demand may have played a part in 4-year olds' apparent lack of sensitivity to second-order relational information. Moreover, because the children in Mondloch et al. were familiarized to two different faces that differed in terms of featural as well as relational information, they may have selectively attended to the featural information (which is thought to 
dominate face processing in early childhood). In contrast, in the current experiments, relational information defined the differences between faces, and this may have cued the infants to attend to this kind of information.

\section{Valentine’s 'Face-Space’ Hypothesis}

Valentine’s (1991) ‘face-space hypothesis’ describes a process in which people form a 'prototype' of an upright face with increasing exposure to upright faces. According to Valentine, faces are represented in a multi-dimensional space and the distance between faces in this space determines the ease of discrimination between them. People recognize faces, according to this theory, by comparing the characteristics of faces that they encounter to a prototype. At the same time, the information from these faces modifies the prototype. Extrapolating Valentine's theory to development, one can posit that, early in life, information in this face-space is most likely meager due to exposure to a limited number of faces. Thus, the dimensions that determine the face-space and the 'prototypical' face may be skewed because they are derived from a limited number of faces. As people gain expertise through exposure to the faces around them, the prototype becomes closer and closer to the norm for this population. De Haan et al. (2001) found that 3-month-olds (but not 1-month-olds) were able to form a prototype of the average of four different normal upright faces. This supports the notion that infants are capable of forming normal upright facial prototypes based on the information in their face-space, although the types of information that infants use in forming these prototypes was not addressed in this study.

Some researchers have referred to the face-space framework in regard to developmental changes in face processing (McKone \& Boyer, 2006; Mondloch et al., 2004; Pellicano \& Rhodes, 2003). Some (Mondloch et al., 2004; Pellicano \& Rhodes, 2003) speculate that adultlike processing of facial information may not be established in infancy or early childhood because lack of experience has resulted in prototypes that are not detailed enough to allow comparisons of faces based on certain dimensions of face processing (according to Mondloch et al., this includes dimensions that involve second-order relational processing). Others (McKone \& Boyer, 2006) suggest that young children potentially have adult-like processing of relational information, but that the results of their study do not conclusively allow them to make this claim. The current study suggests that infants as young as 5 months of age have had enough exposure to upright faces to form a prototype of a normal upright face that includes second-order relational information. It is also clear that the second-order relational information in inverted faces is not 
part of this facial prototype at this age, as evidenced by the clear inversion effect that these infants exhibited when second-order relational information was manipulated. This inversion effect suggests that the information contained in the prototype that infants have of a human face is refined enough to contain adult-like aspects of face processing that are specific to canonical orientation.

Curiously, infants in the Bhatt et al. (2005) study also exhibited an inversion effect, although the stimuli used in that study were line drawings displaying relational manipulations that were outside of the normal human range. This result may indicate that, while infants have a facial prototype that includes information necessary for adult-like processing (i.e., second-order relational information), the prototype may not be refined enough to be bound by normal human face proportions. However, the present study shows that, even with what is perhaps a lessrefined 'face space,' infant's detection of second-order information is good enough to discriminate between less dramatic, more normative second-order alterations as well.

\section{The Uniqueness of Face Processing}

As mentioned in the introduction, one of the issues that is hotly debated in the literature is the uniqueness of face processing. The current study indicates that, by at least 5 months of age, infants have the ability to detect second-order information in faces. This suggests that infants have the tools available to gain expertise with faces at this age (according to Diamond and Carey, 1986). The fact that the 'face-space' of 5-month-olds is sophisticated enough to detect second-order relational information and show an inversion effect suggests that infants do not need exposure to an enormous number of faces in order to form a canonical prototype. Five months is an early age for infants to show the beginning signs of expertise; but it is clear that faces are important to infants and serve a valuable purpose early on. Our study does not directly address the question of the mechanism responsible for the rapid attainment of the tools of expert face processing. Humans may have a specialized section of the brain that is meant for face processing at birth (the Fusiform Face Area), they may be attracted to face-like patterns, or there may be yet another explanation that researchers have not explored as of yet. Despite the fact that a large number of questions remain unanswered, it is clear that faces are important to infants at a very early age and that their perceptual system is constructed in such a way that faces are elevated above most other objects, perhaps all other objects. 


\section{Future Studies}

The present study suggests that 5- and 7-month-old infants are sensitive to second-order relational information. One critical question that follows is: when in life (before 5 months of age) does the ability to process second-order information develop? Bhatt et al. (2005) found that 3-month-olds are insensitive to second-order relational information when shown line drawings with exaggerated manipulations of the second-order relational information. This suggests that the ability to process second-order information develops sometime between 3 and 5 months of age. However, infants younger than 5 months of age may perform differently on photographs of faces; therefore, future studies need to examine younger infants' performance on photographs using procedures of the sort used in the current experiments.

If the ability to process second-order information does develop sometime between birth and 5 months of age, such that infants younger than a certain age are insensitive to second-order information, then the question arises as to the nature of the mechanism(s) that drive this developmental change. Is experience with a varied number of faces the primary instigator of second-order processing? In addition, does the limited population of faces that infants are exposed to early in life differentially affect the ability to process second-order information? For instance, because infants are more likely to have female than male caregivers with whom they have extensive contact (Quinn, Yahr, Kuhn, 2002), does it mean that infants are more adept at processing second-order information in female than in male faces?

Other questions concerning the nature of development of second-order relational information pertain to the perceptual mechanisms that change with development. For instance, are perceptual limitations of younger infants, such as the inability to process fine spatial information such as distances between features, the reason for younger infants' lack of ability to process second-order information? Are there attentional mechanisms that are also involved? For instance, can the salience of the relational information be enhanced for younger infants? Future studies will need to answer these and other such questions that address the development of second-order relational information processing, which underlies the highly significant socialcognitive function of face processing. 
Table 7.1 Overview of all experiments

\begin{tabular}{|c|c|c|c|c|}
\hline Experiment & $\begin{array}{l}\text { Age of } \\
\text { Infants }\end{array}$ & Paradigm & $\begin{array}{l}\text { Face } \\
\text { Manipulation }\end{array}$ & Results \\
\hline One & 7 months & Paired Comparison & Upright faces & No preference \\
\hline Two & 7 months & Habituation & Upright faces & $\begin{array}{l}\text { Suggestion of discrimination } \\
\text { with habituation to the } \\
\text { normal face. }\end{array}$ \\
\hline Three & 7 months & $\begin{array}{l}\text { motion-enhanced } \\
\text { discrimination } \\
\text { procedure }\end{array}$ & Upright faces & $\begin{array}{l}\text { Discrimination in all } \\
\text { conditions- preference for } \\
\text { non-moving face. }\end{array}$ \\
\hline Four & 5 months & $\begin{array}{l}\text { motion-enhanced } \\
\text { discrimination } \\
\text { procedure }\end{array}$ & $\begin{array}{l}\text { Upright and } \\
\text { inverted faces }\end{array}$ & $\begin{array}{l}\text { Discrimination in all upright } \\
\text { conditions- preference for } \\
\text { non-moving face. } \\
\text { No discrimination in the } \\
\text { inverted conditions. } \\
\text { Suggestion of an inversion } \\
\text { effect. }\end{array}$ \\
\hline
\end{tabular}

Copyright (C) Angela Hayden 2006 


\section{References}

Bartlett, J. \& Searcy, J. (1993). Inversion and configuration of faces. Cognitive Psychology, 25, 281-316.

Bentin S., Allison, T., Puce, A., Perez, E., \& McCarthy, G. (1996). Electrophysiological studies of face perception in humans. Journal of Cognitive Neuroscience, 8, 551-565.

Bertin, E., \& Bhatt, R.S. (2004). The Thatcher illusion and face processing in infancy. Developmental Science, 4, 423-432.

Bhatt, R.S., Bertin, E., Hayden, A., \& Reed, A. (2005). Face processing in infancy: developmental changes in the use of different kinds of relational information. Child Development, 76(1), 169-181.

Bhatt, R.S., Hayden, A., Reed, A., Bertin, E., \& Joseph, J. (2006). Infants’ perception of information along object boundaries: Concavities versus convexities. Journal of Experimental Child Psychology, 94(2), 91-113.

Brace, N.A., Hole, G.J., Kemp, R.I., Pike, G.E., Van Duuren, M., \& Norgate, L. (2001). Developmental changes in the effect of inversion: Using a picture book to investigate face recognition. Perception, 30(1), 85-94.

Carey, S. \& Diamond, R. (1994). Are faces perceived as configurations more by adults than by children? Visual Cognition, 1 (2/3), 253-274.

de Haan, M., Johnson, M.H., Maurer, D., \& Perrett, D.I. (2001). Recognition of individual faces and average face prototypes by 1- and 3- month-old infants. Cognitive Development, 16(2), 659-678.

de Schonen, S., \& Mathivet, E. (1989). First come first served: a scenario about the development of hemispheric specialization in face processing in infancy. European Bulletin of Cognitive Psychology, 9, 3-44.

DeLoache, J., Pierroutsakos, S., \& Uttal, D. (2003). The origins of pictorial competence. Current Directions in Psychological Science, 12, 114-118.

Diamond, R, \& Carey, S. (1977). Developmental changes in the representation of faces. Journal of Experimental Child Psychology, 23, 1-22.

Diamond, R., \& Carey, S. (1986). Why faces are and are not special: An effect of expertise. Journal of Experimental Psychology: General, 115(2), 107-117. 
Farkas, L.G. (1994). Anthropometry of the head and face in medicine (2nd ed.). New York: Elsevier.

Farah, M.J., Rabinowitz, C., Quinn, G.E., \& Liu, G.T. (2000). Early commitment of neural substrates for face recognition. Cognitive Neuropsychology, 17, 117-123.

Felician, O., Ceccaldi, M., Didic, M., Thinus-Blanc, C. \& Poncet, M. (2003). Pointing to body parts: A double dissociation study. Neuropsychologia, 41, 1307-1316.

Freire, A., Lee, K., \& Symons, L.A. (2000). The face-inversion effect as a deficit in the encoding of configural information: direct evidence. Perception, 29, 159-170.

Gauthier, I. \& Curby, K.M. (2005). A perceptual traffic jam on highway N170: Interference between face and car expertise. Current Directions in Psychological Science, 14(1), 3033.

George, N., Evans, J., Fiori, N., Davidoff, J., \& Renault, B. (1996). Brain events related to normal and moderately scrambled faces. Cognitive Brain Research, 4, 65-76.

Gilchrist, A., \& McKone, E. (2003). Early maturity of face processing in children: Local and relational distinctiveness effects in 7-year-olds. Visual Cognition, 10(7), 769-793.

Goldstein, A.G., \& Chance, J.E. (1964). Recognition of children’s faces. Child Development, 35, 129-236.

Goren, C.C., Sarty, M. \& Wu, P.K.Y. (1975). Visual following and pattern discrimination of face-like stimuli by newborn infants. Pediatrics, 56, 544-549.

Haith, M.M., \& McCarty, M.E. (1990). Stability of visual expectations at 3.0 months of age. Developmental Psychology, 26(1), 68-74.

Hay, D.C., \& Cox, R. (2000). Developmental changes in the recognition of faces and facial features. Infant and Child Development, 9, 199-212.

Itier, R.J., \& Taylor, M.J. (2004). Effects of repetition and configural changes on the development of face recognition processes. Developmental Science, 7(4), 469-487.

Jiang, X., Rosen, E., Zeffiro, T., VanMeter, J., Blanz, V., \& Riesenhuber, M. (2006). Evaluation of a shape-based model of human face discrimination using fMRI and behavioral techniques. Neuron, 50, 159-172.

Johnson, M.H., \& Morton, J. (1991). Biology and cognitive development: The case of face recognition. Oxford, UK: Blackwell. 
Kanwisher, N., McDermott, J., \& Chun, M.M. (1997). The fusiform face area: A module in human extrastriate cortex specialized for face perception. Journal of Neuroscience, 17(11), 4302-4311.

Leder, H. (1996). Line drawings of faces reduce configural processing. Perception, 25(3), 355-366.

Leder, H. (1999). Matching person identity from facial line drawings. Perception, 28(9), 11711175.

Lewis, M.B. (2003). Thatcher's children: development and the Thatcher illusion. Perception, 32, 1415-1421.

McKone, E. (2004). Isolating the special component of face recognition: Peripheral identification and a Mooney face. Journal of Experimental Psychology: Learning, Memory, and Cognition, 30(1), 81-97.

McKone, E., \& Boyer, B.L. (2006). Sensitivity of 4-year-olds to featural and second-order relational changes in face distinctiveness. Journal of Experimental Child Psychology, 94(2), 134-162.

Mondloch, C.J., Dobson, K.S., Parsons, J., \& Maurer, D. (2004). Why eight-year-olds cannot tell the difference between Steve Martin and Paul Newman: Factors contributing to the slow development of sensitivity to the spacing of facial features. Journal of Experimental Child Psychology, 89, 159-181.

Mondloch, C.J., Le Grand, R., \& Maurer, D. (2002). Configural face processing develops more slowly than featural face processing. Perception, 31, 553-566.

Mondloch, C.J., Leis, A., \& Maurer, D. (2006). Recognizing the face of Johnny, Suzy, \& me: Insensitivity to the spacing among features at 4 years of age. Child Development, 77(1), 234-243.

Moscovich, M., \& Moscovich, D.A. (2000). Super face-inversion effects for isolated internal or external features, and for fractured faces. Cognitive Neuroscience, 17, 201219.

Murray, J.E., Yong, E., \& Rhodes, G. (2000). Revisiting the perception of upside-down faces. Psychological Science, 11(6), 492-496. 
Pascalis, O., de Schonen, S., Morton, J., Deruelle, C., \& Fabre-Grenet, M. (1995).

Mother's face recognition by neonates: a replication and extension. Infant Behavior and Development, 18, 79-85.

Pellicano, E., \& Rhodes, G. (2003). Holistic processing of faces in preschool children and adults. Psychological Science, 14(6), 618-622.

Pellicano, E., Rhodes, G., \& Peters, M. (2006). Are preschoolers sensitive to configural information in faces? Developmental Science, 9(3), 270-277.

Quinn, P.C., Yahr, J., \& Kuhn, A. (2002). Representation of the gender of human faces by infants: a preference for female. Perception, 31, 1109-1121.

Riesenhuber, M., Jarudi, I., Gilad, S., \& Sinha, P. (2004). Face processing in humans is compatible with a simple shape-based model of vision. Proceedings of the Royal Society of London B (suppl), 271, S448-S450.

Rose, S.A., Jankowski, J.J., \& Feldman, J.F. (2002). Speed of processing and face recognition at 7 and 12 months. Infancy, 3(4), 435-455.

Schwartzer, G. (2002). Processing of facial and non-facial visual stimuli in 2-5-year-old children. Infant and Child Development, 11(3), 253-270.

Schwarzer, G. \& Zauner, N. (2003). Face processing in 8-month-old infants: Evidence for configural and analytical processing. Vision Research, 43, 2783-2793.

Searcy, J.H., \& Barlett, J.C. (1996). Inversion and processing of component and spatialrelational information in faces. Journal of Experimental Psychology: Human Perception and Performance, 22, 904-915.

Slaughter, V., \& Heron, M. (2004). Origins and early development of human body knowledge. Monographs of the Society for Research in Child Development, 69(2, Serial No. 276).

Thompson, L.A., Madrid, V., Westbrook, S., \& Johnston, V. (2001) Infants attend to second- order relational properties of faces. Psychonomic Bulletin and Review, 8(4), 769777.

Turati, C. (2004). Why faces are not special to newborns: an alternative account of the face preference. Current Directions in Psychological Science, 13(1), 2004, 5-8.

Tversky, B., \& Baratz, D. (1985). Memory for faces: are caricatures better than photographs? Memory and Cognition, 13(1), 45-49. 
Valentine, T. (1991). A unified account of the effects of distinctiveness, inversion, and race in face recognition. The Quarterly Journal of Experimental Psychology A: Human Experimental Psychology, 43A(2), 161-204.

Walton, G.E., Bower, N.J.A., \& Bower, T.G.R. (1992). Recognition of familiar faces by newborns. Infant Behavior and Development, 15, 525-535.

Wilson, R.S., Kasniak, A.W., Klawans, H.L., \& Garron, D.C. (1980). High speed memory scanning in Parkinsonism. Cortex, 16(1), 67-72.

Yin, R.K. (1969). Looking at upside-down faces. Journal of Experimental Psychology, 81, 141145.

Yovel, G., \& Duchaine, B. (2006). Specialized face perception mechanisms extract both part and spacing information: Evidence from developmental prosopagnosia. Journal of Cognitive Neuroscience, 18(4), 580-593.

Copyright (C) Angela Hayden 2006 


\section{Vita}

Angela Hayden

Born: 8/17/81 in Louisville, KY

\section{$\underline{\text { Education }}$}

University of Kentucky, (Ph.D. program)

Program: Cognitive/Developmental Psychology $\quad$ August, 2004 - Present

University of Kentucky, B.A. (magna cum laude) August, 1999 - May, 2003

Major: Psychology

\section{$\underline{\text { Professional Positions Held }}$}

Laboratory Technician, University of Kentucky $\quad$ May 2003 - August 2004

Research Assistant, University of Kentucky July 2004 - Present

Teaching Assistant, University of Kentucky August 2006 - Present

$\underline{\text { Scholastic and Professional Honors }}$

\section{Fellowships}

Departmental Fellowship, Department of Psychology $\quad$ August 2004 - August 2005:

Travel Award

April 2005

Awards:

Academic Excellence Scholarship

August 1999 - May 2003

Bellsouth National Merit Scholarship

August 1999- May 2000

\section{$\underline{\text { Professional Publications }}$}

\section{Journal Articles}

Bhatt, R.S., Bertin, E., Hayden, A. \& Reed, A. (2005). Face processing in infancy:

Developmental changes in the use of different kinds of relational information. Child Developmental, 76, 169-181. 
Bhatt, R.S., Hayden, A., Reed, A., Bertin, E. \& Joseph, J. (2006). Infants’ perception of information along object boundaries: Concavities versus convexities. Journal of Experimental Child Psychology, 93, 91-113.

Hayden, A., \& Bhatt, R.S. (in press). Infants' sensitivity to uniform connectedness as a cue for perceptual organization. Psychonomic Bulletin and Review.

Hayden, A., Bhatt, R.S., Reed, A., \& Joseph, J. (in press). The other-race effect in infancy: Evidence using a morphing technique.

\section{Conference Posters}

Bhatt, R., Bertin, E., Hayden, A. (2005). Face processing in infancy: Developmental changes in the use of different kinds of relation information. Presented at the biennial meeting of the International Society for Infant Studies in Chicago, Illinois, May 5-8. (poster presentation)

Hayden, A., Bhatt, R.S., \& Quinn, P.C. (2005). Luminance but not shape similarity dominated uniform connectedness as a perceptual organizational cue in infancy. Presented at the biennial meeting for the Society for Research in Child Development meeting, Atlanta, Georgia, April . (poster presentation)

Reed, A.W., Hayden, A., Bertin, E., \& Bhatt, R.S. (2005). Face processing in infancy: Further evidence of sensitivity to second-order relational information. Presented at the biennial meeting for the Society for Research in Child Development, Atlanta, Georgia, April 7-10. (poster presentation)

Reed, A.W., Hayden, A., \& Bhatt, R.S. (2005). Expectancy-based behavior in infancy: Convergent evidence using a new procedure. Presented at the biennial meeting for the Society for Research in Child Development, Atlanta, Georgia, April 7-10. (poster presentation).

\section{Angela Hayden}

